\title{
Monte-Carlo Parallel Simulation of Phonon Transport for 3D Nano-Devices
}

\author{
Zahra Shomali $^{\mathrm{a}}$, Behrad Pedar ${ }^{\mathrm{a}}$, Jafar Ghazanfarian ${ }^{\mathrm{b}, *}$, Abbas Abbassi $^{\mathrm{a}}$ \\ ${ }^{a}$ Department of Mechanical Engineering, Amirkabir University of Technology, Tehran, \\ Iran. \\ ${ }^{b}$ Department of Mechanical Engineering, Faculty of Engineering, University of Zanjan, \\ University Blvd. 45371-38791, Zanjan, Iran.
}

\begin{abstract}
Due to the importance of the transistors in nano-electronics technology, the accurate study of these nano-devices is an essential field of research. Taking into account the non-Fourier nature of heat transfer with considering the actual three-dimensional structure of the MOSFETs, are the challenges in transistor analysis which have not been studied precisely yet. Using the Monte-Carlo method for solving the Boltzmann equation, two actual threedimensional silicon transistors are accurately simulated. First, the accuracy of the method is verified by the investigation of heat transport in different regimes for a cuboid. Then, the procedure is used to simulate a 2-D silicon MOSFET. The obtained results present good consistency with the existent data. Then, the 3-D MOSFET is investigated in two different cases with various boundary conditions, and the formation of the hot zone is announced. Farther, it is attained that a temperature-jump occurs around the time $t=22 \mathrm{ps}$. This phenomena is attributed to the change of the type of
\end{abstract}

\footnotetext{
*Corresponding author. Tel.: +98 2433054142

Email address: j.ghazanfarian@znu.ac.ir (Jafar Ghazanfarian )
} 
the dominant heat carriers from acoustic phonons to the optical ones. Furthermore, it is found that although the transistor is still heated the peak temperature starts to decrease when time passes $t=200 \mathrm{ps}$. Moreover, as the heat source is switched off at $t=500 \mathrm{ps}$, the temperature is acquired to suffer many fluctuations until the expecting descending behaviour appears at $t=726 \mathrm{ps}$. Such deportment takes place due to the imprisoning the existent heat in the hot-spot between the isolated boundaries. Besides, the results reveals that redesigning the transistors in case two by leaving the end parts of the top boundary which are in contact with the metallic material open, strengthens the presence of the hot-spot while fastening the heat release to the environment. Also the temperature profiles of the second case exhibits odd behaviours as well. Finally, it should be emphasized that this research compensates the lack of heat transfer data for the thermal investigation of 3-D MOS devices.

Keywords: Monte-Carlo, Nanoscale, MOS Devices, Phonon Transport, Boltzmann Equation 


\section{1. Introduction}

2

3

4

5

6 7 should be considered [1].

8

The MOS field-effect-transistors are the main blocks of the nano-electronic

technology. Nowadays, due to the aim toward more compact, faster, and stronger processors, accurate simulation of what occurs in these vital nanodevices is indispensable. Strictly speaking, the real three-dimensional structure of the transistors with the actual thermal and electrical circumstances

As the size of the electronic devices shrinks, the use of the prevailing methods like the Fourier's law leads to the wrong predictions. This is due to the appearance of the non-Fourier effects for the systems with dimensions below the micrometer [2]. There are several kinds of methods for the study of non-Fourier heat conduction. From one hand, these procedures include atomistic-statistic methods such as molecular dynamics or methods based on the semi-classical Boltzmann transport equation (BTE) for phonons. On the other hand it contains macroscopic continuum models like single phaselag (SPL) [3], dual-phase-lag (DPL) [4, 5, 6] or the thermo-mass theory [7]. Macroscopic models are usually phenomenological methods which need verification for the calculation of coefficients. This requirement, makes the mesoscopic methods, the imperative tool for nano-scale transient heat conduction 
modeling, which provide the requisite data for corroboration the simple continuum techniques.

The methods which deal with solving the BTE $[8,9,10,11,12,13,14]$ have taken much attention for a long time. As long as phonons can be considered to be particles, or on other words, the quantum wave effects are neglected, the Boltzmann transport equation can be used to model the heat conduction phenomenon. There are two techniques for obtaining the numerical solution of the BTE. One is the deterministic approach, and the other is the stochastic Monte-Carlo (MC) [15] method. Stochastic method, which is also the interest of this paper, usually is used to solve the partial differential equations that involve a large number of independent variables.

Peterson [16] was the first who presented the Monte-Carlo procedure for investigation of the phonon transport. In the work by Peterson, the phonon dispersion and the various phonon polarizations were not considered. The studies by Mazumder and Majumdar [17, 18] have resolved these failures. The consideration of optical phonon modes on thermal conductivity calculation was suggested by Narumanchi et al. [19] and Wang [20]. Narumanchi and co-workers applied two assumptions while taking into account optical phonons. The group velocity of optical phonons is taken to be zero and also, 
no distinction is made between transverse optical (TO) and longitudinal optical (LO) modes. Wang resolved the first simplifier assumption. While the second postulation was rectified with contemplation of the role of various optical phonon modes on thermal conductivity by Mittal and Mazumder [21]. Also, Wong et al. [22] were the first who entered the concept of internal heat generation with simple procedure to the MC simulation. Nowadays, it is a usual and correct method to model the electron and phonon transport separately and this modeling is proven to present acceptable results.

As the Monte-Carlo method uses the physics of phonons, such as, phonon dispersion and phonon scatterings in simulation of the phonon transport, it is confirmed qua an accurate and flexible method. Also, the MC method linearly scales with the number of dimensions and can be used to model complex geometries. Despite these advantages, the MC method is computationally expensive and is predisposed to statistical variations. There have been some efforts to overcome these disadvantages like the steady-state approach which was suggested by Randrianalisoa and Bailis [23] to reduce the computational time and memory for calculation of thermal conductivity of thin films. Also, Hadjiconstantinou et al. [24] presented the variance-reduced 
stochastic particle simulation method for solving the relaxation-time model of the BTE that results in an efficient computational cost. Nonetheless, until now, the use of the Monte-Carlo method has been limited to almost simple geometries.

This stochastic Monte-Carlo method with different procedures has been used to predict the thermal conductivity of silicon nano-wires [25, 26], model the phonon transport in porous silicon [27, 28], and other transistor candidate compounds [29], and simulate the heat generation process in silicon MOSFETs [30, 31, 32, 33]. Besides, in contrast to the use of the Monte-Carlo method for two-dimensional nano-devices [34, 35].

To the best of our knowledge, the transient heat conduction in 3-D nanodevices is an almost untouched subject of interest. Specifically, there have been no molecular dynamic nor Monte-Carlo investigation of these threedimensional systems. In the present paper, the Monte-Carlo method is used to study the transient heat conduction in 3-D silicon MOSFETs. The concept of heat generation is entered by the method used in Ref. [22]. The heating and cooling procedures are investigated for two different cases of 3-D silicon MOSFETs with different boundary conditions. This study fulfills the gap existed in the field of 3-D transient heat conduction modeling, and supports 
77

78

79

80

81

82

83

84

85

86

87

88

89

90

91

92

93

94

the intransitive data for phenomenological macroscopic calculations.

In this work, in Sec. 2, the geometry and the conditions of the simulated MOSFET are given. The mathematical considerations are presented in Sec. 3. Also, Sec. 4 is involved with the numerical method inspection. Then in Sec. 5, the accuracy of the written three-dimensional code is checked by verification the obtained results with the existent data of a cuboid and a 2-D MOSFET. Finally, the results of the simulation of the 3-D silicon MOSFET with two different boundary conditions are dispensed in 6 .

\section{Geometry and boundary conditions}

The main goal of this paper is performing the non-Fourier Monte-Carlo investigation of the transient heat conduction in SOI three-dimensional MOSFETs. Two types of structures with different boundary conditions are investigated. The heat source and boundary conditions are similar to what exists in a real 3-D MOSFET nano-device. As it is seen in Fig. 1(a), the First case is a three-dimensional common MOSFET nano-device in which the existence of the thick layer of $\mathrm{SiO}_{2}$ and $\mathrm{Si}$ substrates under the silicon channel is presented by the boundary conditions. The front, back, left, right, and top boundaries are assumed to be adiabatic according to the low conductivity 
of the thick isolating oxide covering the surfaces. The bottom boundary is exposed to the ambient temperature while the temperature jump is taken into account. This boundary condition is named as the open boundary condition. It is assumed that the initial temperature in all parts of the MOSFET is $299 \mathrm{~K}$.

It is important to note that the most part of the heat generated inside the transistor is conducted toward the bottom and then it is dissipated to the surrounding after passing through the bulk silicon. On the other side, the heat flow through the metallic contacts located on top of transistor cannot supposed to be zero. Consequently, the conditions of the second sample is the same as that of case one, with the difference that the two metallic ending parts of the top surface are also exposed to the ambient temperature.

This is an accurate consideration as the metallic contacts are the excellent heat conductors. This open boundary condition helps to more realizing of the modeling. The sizes of the transistors in our simulation are $L_{x}=100 \mathrm{~nm}, L_{y}=1 \mu \mathrm{m}$, and $L_{z}=50 \mathrm{~nm}$, respectively, in $X, Y$, and $Z$ directions with the heat generation area of $10 \mathrm{~nm} \times 1 \mu \mathrm{m} \times 10 \mathrm{~nm}$ [36]. It should be noted that only below $\mathrm{t}=\mathbf{5 0} \mathrm{ps}$, the geometry can be considered as a 2-D transistor since $1 \mu \mathrm{m}$ in the third length 


\section{direction is much longer than the phonon traveling length $30 \mathrm{~nm}$ in}

10 ps. In the work by Sverdrup and Goodson [37], the heat generation rate caused by strong electron-phonon scattering is calculated via the electrical simulation in MEDICI. As the heat generation is peaked on the drain side of the transistor [37], the region of heat generation is also confined to this zone heading to a localized heat source. Consequently, to keep away from electro-thermal coupling, the heat generation rate is modeled with the nonuniform heat generation profile of $Q_{\max }(X+Z-1)$ accompanied by $Q_{\max }=$ $10^{19} \mathrm{~W} / \mathrm{m}^{3}$. This heat flux is applied for all values of $\mathrm{y}$-coordinate [38].

\section{Mathematical modeling}

Phonons are the main heat carriers in silicon. As the characteristic length and the time scale are, respectively, larger than the phonon coherence length and longer than the phonon relaxation time, the phonons can be treated as semiclassical particles. Hence, the Boltzmann transport equation is used to govern these phonons via describing the phonon distribution function, $f_{b}(\mathbf{r}, \mathbf{q}, \mathbf{t})$. This distribution depends on the location $\mathbf{r}$, the wave vector $\mathbf{q}$, and the time $t$ for each phonon branch, $b$. The phonon Boltzmann transport equation which includes both equilibrium and non-equilibrium phenomena is 


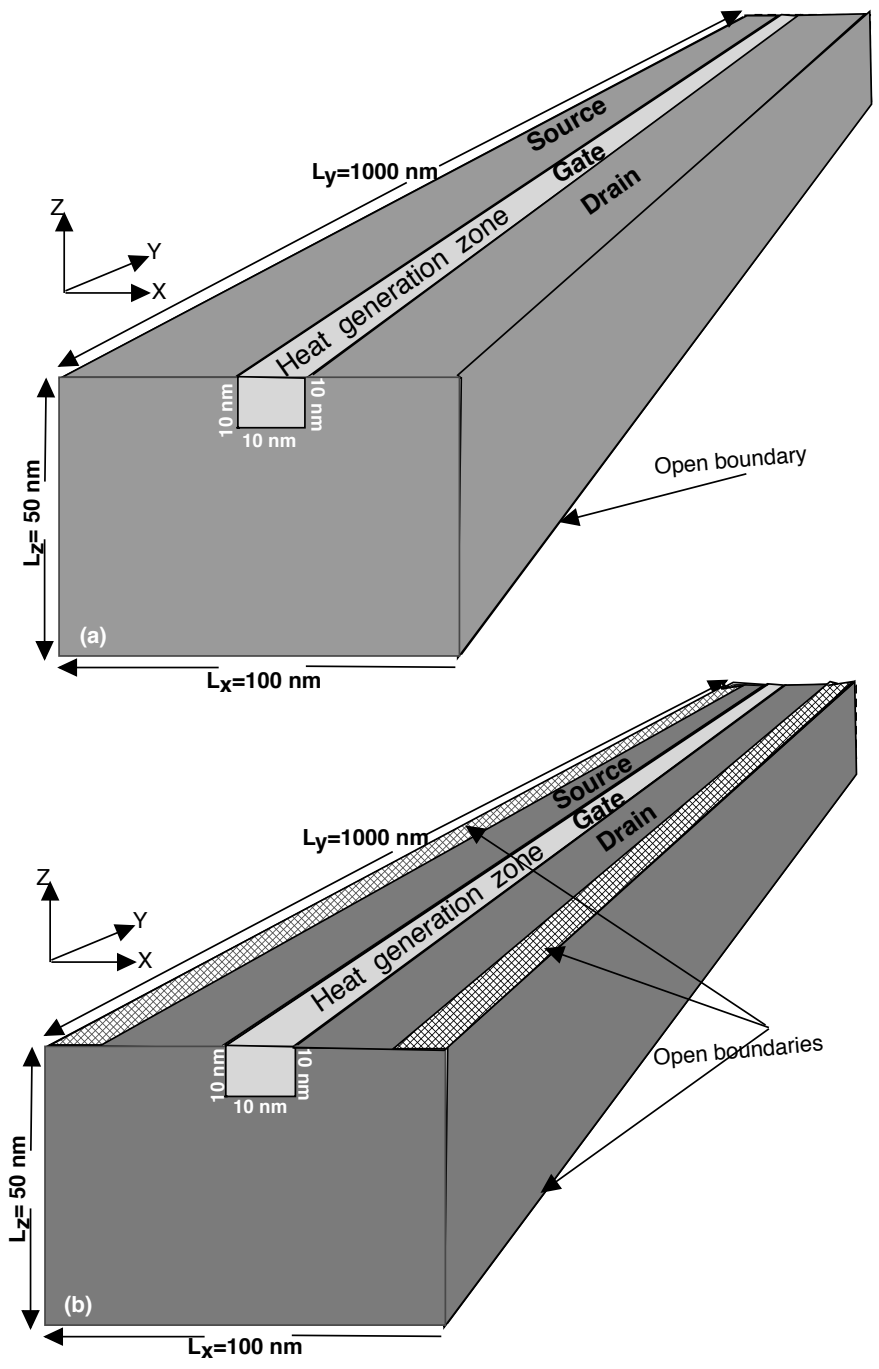

Figure 1: (a) Case one: the 3-D silicon MOSFET with bottom open boundary condition. (b) Case two: the 3-D transistor with two top and one bottom open boundaries. All other boundaries are adiabatic for both cases. 
written as

$$
\frac{\partial f_{b}(\mathbf{r}, \mathbf{q}, t)}{\partial t}+v_{b, \mathbf{q}} \cdot \nabla_{r} f_{b}(\mathbf{r}, \mathbf{q}, t)=\left.\frac{\partial f_{b}(\mathbf{r}, \mathbf{q}, t)}{\partial t}\right|_{s c a t} .
$$

133

${ }_{144}<f_{B E}>[39]$.

In equation $1, v_{b, \mathbf{q}}=\frac{d \omega_{b, \mathbf{q}}}{d q}$ is the group velocity of phonons where $\omega_{b, \mathbf{q}}$ is the angular frequency of branch $b$ with the wave vector q. In general, the frequency depends on both the value and the direction of the wave-vector. With isotropic assumption, the frequency only reliance on the value of the wave-vector and remains same for all directions of the space. In this paper, each phonon branch is treated with the isotropic approximation. Hence, the phonon dispersion relation of the silicon MOSFET will be considered isotropic. Here, the stochastic phonon Monte-Carlo method is used to solve the PBTE. Modeling with the PMC starts with considering the equilibrium condition. The phonon distribution function at the absolute temperature $T$, in the equilibrium situation is the famous Bose-Einstein (BE) distribution, In the MC method, phonons considered as statistical samples are assigned to six individual stochastic spaces: three wave vector and three position vector components. The sampled phonons first undergo drift (ballistic motion) 
and then, are submitted to the scattering processes. The steps of MC method for simulating the 3-D silicon MOSFET come in the following section.

\subsection{Number of Stochastic Samples}

First, the number of phonons per unit volume that are present in the computational domain, $N_{\text {actual }}$, should be calculated. In the work by Mazumder and Majumdar [17], the Bose-Einstein density function is measured from $0 K$. So, as an instance, in a computational space in which the minimum available temperature is $300 \mathrm{~K}$, there are many phonons that are not involved in the heat transfer process, but they only exist to convey the temperature to $300 \mathrm{~K}$. This large amount of phonons takes too much memory and makes the computation needlessly complex. In the present research, in the continuance of the work by Wong et al. [22], a reference temperature, $T_{r e f}$, which is the minimum temperature in the solution domain is introduced. Consequently, as the initial temperature of the sample is taken equal to the reference temperature, the real number of the phonons is zero in the initial condition. Hence, the actual number of phonons is computed as,

$$
N_{\text {actual }}=\sum_{p} \int\left[f_{B E}(\omega, T)-f_{B E}\left(\omega, T_{r e f}\right)\right] \frac{|\mathbf{K}|^{2}}{2 \pi^{2}}
$$




$$
\left.\frac{d|\mathbf{K}|}{d \omega}\right|_{\omega} d \omega
$$

Here, $\left.\frac{|\mathbf{K}|^{2}}{2 \pi^{2}} \frac{d|\mathbf{K}|}{d \omega}\right|_{\omega}$ or $D(\omega)$ is the phonon density of states. The integration is down over the whole frequency space for each phonon polarization. The integration is numerically performed by discretizing the frequency space. The maximum frequency in each branch, $\omega_{\max }$, is driven from each dispersion curve. The frequency interval between 0 and $\omega_{\max }$ are divided into the $N_{b}$ intervals with $\omega_{c, i}=\frac{2 i-1}{2} \Delta \omega_{i}$ and $\Delta \omega_{i}$ being the central frequency and the bandwidth of the i-th spectral bin, respectively. This reduces the integral into a summation over discrete frequency intervals,

$$
\begin{array}{r}
N_{\text {actual }}=\sum_{p} \sum_{i=1}^{N_{b}}\left[f_{B E}\left(\omega_{c, i}, T\right)-f_{B E}\left(\omega_{c, i}, T_{r e f}\right)\right] \frac{|\mathbf{K}|^{2}}{2 \pi^{2}} \\
\left.\frac{d|\mathbf{K}|}{d \omega}\right|_{\omega_{c, i}} \Delta \omega_{i} .
\end{array}
$$

In this paper, $\Delta \omega_{i}$ is taken to be $10^{11}$ and $N_{b}$ is 1000 . It is shown that for a 3-D silicon, these values of parameters guarantee the accurateness of the solutions. The actual number of phonons obtained from Eq. 3 is usually a very large number which makes the modeling to be computationally expensive. Hence, by introducing the scaling or a weighting factor, $W$, the number 
177 of phonons which are simulated, are decreased:

$$
W=\frac{N_{\text {actual }}}{N_{\text {prescribed }}}
$$

178

$N_{\text {prescribed }}$ is the number of phonons (stochastic samples) actually initialized/emitted into the system. A same weighting factor, $W$, is used for each branch.

\subsection{Initialization of Phonon Samples}

\subsubsection{Position}

The simulation domain is a three-dimensional silicon cube of width $L_{x}$, length $L_{y}$, and height $L_{z}$. The sample phonons are spatially initialized by dividing the computational domain into a number of cells or control volumes. In 3-D simulations, the control volume is usually taken to be a cube. Hence, three random numbers, $R_{1}, R_{2}$ and $R_{3}$, satisfying $0<R_{1}, R_{2}, R_{3}<1$, are sufficient to determine the coordinates of the phonon ensembles inside the control volumes. the position vector is obtained as

$$
\vec{r}=R_{1} L_{x} \hat{x}+R_{2} L_{y} \hat{y}+R_{3} L_{z} \hat{z}
$$


Just, initializing the position of the phonon, the frequency should be sampled.

The next subsection involves with determining the frequency.

\subsubsection{Frequency}

The frequency of the phonon ensembles are sampled by calculating the number of phonons in the i-th interval of frequency, $\omega_{i}$. With due attention

to the Eq. 3, the number of phonons in the i-th spectral frequency bin can be written as:

$$
N_{i}=\left.\sum_{p}\left(\frac{1}{\exp \left(\frac{\hbar \omega_{c, i}}{k_{B} T}\right)-1}-\frac{1}{\exp \left(\frac{\hbar \omega_{c, i}}{k_{B} T_{r e f}}\right)-1}\right) \frac{|\mathbf{K}|^{2}}{2 \pi^{2}} \frac{d|\mathbf{K}|}{d \omega}\right|_{\omega_{c, i}} \Delta \omega_{i} .
$$

Consequently, a normalized frequency cumulative density function, $C D F$ is constructed,

$$
F_{i}=\frac{\sum_{j=1}^{i} N_{j}}{\sum_{j=1}^{N_{b}} N_{j}} .
$$

Here, $F_{i}$ shows the probability of finding a phonon with frequency less than $\omega_{c, i}+\Delta \omega_{i}$. As a result, $F_{i}-F_{i-1}$ is the probability of finding a phonon in the i-th frequency interval. For sampling the frequency of the phonon, a random number $R_{F}$, between zero and one, is drawn. Then by using the bisection algorithm, the $\mathrm{i}$-th frequency bin in which the relation $F_{i-1}<R_{F}<F_{i}$ is satisfied, will be calculated. So, the initial sampled frequency is determined 
205

via

$$
\omega=\omega_{c, i}+\left(2 R_{F}-1\right) \frac{\Delta \omega_{i}}{2} .
$$

\subsubsection{Polarization}

After a frequency is assigned to the sampled phonon ensemble, another random number must be introduced to select a polarization, $p$, or the branch $b$. The probability of belonging the frequency to the polarization $p$, in the i-th spectral frequency bin is given by

$$
P_{i, p}=\frac{N_{i, p}}{N_{i}}+P_{i, p-1}
$$

where, $N_{i, p}=f_{0}\left(\omega_{c, i}, p\right) D\left(\omega_{c, i}, p\right)$ is the number of phonons in branch $b$ numbered by $p$ in the i-th frequency interval. Also, $N_{i}$ is the total number of phonons in the i-th spectral frequency bin corresponding to the all branches. It should be mentioned that the transverse modes are degenerate and themselves have two branches. It means there are two $T A$ and $T O$ branches. For example the probability of the existence of the LA phonon in the i-th spectral interval can be calculated as[40, 17]: 


$$
\begin{aligned}
P_{i}(L A)= & f_{0}\left(\omega_{c, i}, L A\right) D\left(\omega_{c, i}, L A\right) \frac{1}{f_{0}\left(\omega_{c, i}, L A\right) D\left(\omega_{c, i}, L A\right)+2 f_{0}\left(\omega_{c, i}, T A\right) D\left(\omega_{c, i}, T A\right)} \ldots \\
& \frac{1}{+f_{0}\left(\omega_{c, i}, L O\right) D\left(\omega_{c, i}, L O\right)+2 f_{0}\left(\omega_{c, i}, T O\right) D\left(\omega_{c, i}, T O\right)}
\end{aligned}
$$

As the probability of the polarization $p$ is calculated, a random number, $R_{p}$ is taken into account. If the relation $P_{i-1}<R_{p}<P_{i}$ is established, then the phonon in frequency interval $i$ is ascribed to the polarization $p$ or branch $b$. With $p=1,2, \ldots$ representing different $b$ branches of the dispersion relation and assuming $P_{i, 0}=0$, the procedure explained is quite general. In the present work, two acoustic and two optical branches of silicon are considered. The branches of the sampled phonons frequency are classified as, $L A, T A, L O$ and $T O$ respectively, for the conditions $R_{P}<P(L A)$, $P(L A)<R_{P}<P(T A), P(T A)<R_{P}<P(L O)$, and $R_{P}>P(L O)$. These inputs of the Boltzmann equation are obtained for the present 3-D silicon MOSFET and is represented in Appendix 8. The calculated actual number of the phonons, the phonon distribution in frequency space at $300 \mathrm{~K}$, and the cumulative density of function $F$, for the present study are presented in Figs. 18(a-c) of Appendix. Also, the probability of finding the phonon 
in each of the branches is shown in Fig. 18(d). Once the frequency and polarization of the phonon are obtained, the value of its group velocity is calculated via $v_{g}=\nabla_{k} \omega$. The Fig. 19 shows the acquired group velocity for all branches. In the last step of initialization, the direction is attributed to the each phonon sample, the procedure is the same as the one described in [40].

\subsection{Phonon dispersion}

The phonon dispersion curves present the relation between the angular frequency $\omega$ and the wave vector $q$ in the first Brillouin Zone (1BZ). These curves reflect the symmetry of the underlying lattice. From dispersion, the properties such as group velocity, and density of states can be extracted and used in the solution of the BTE. Here, isotropic relationships is used to approximate the dispersion. Silicon has four phonon polarizations that are two degenerate acoustic branches named as transverse acoustic (TA) and longitudinal acoustic (LA). Also there are two optical branches: transverse optical (TO) and longitudinal optical (LO). The previous studies have confirmed that the contribution from optical branches is notable and should be accounted along the acoustic branches [19, 21].

Here, as the same as done in [41], the quadratic fitting of the 
full dispersion in the (100) crystal direction of the silicon is used to obtain an approximate isotropic relation for the dispersion of the each acoustic and optical branches. Hence, the relation $\omega_{b}=c_{b} k^{2}+v_{b} k+\omega_{0, b}$ with $c_{L A}=-2.00 \times 10^{-7}, c_{T A}=-2.26 \times 10^{-7}, c_{L O}=-1.6 \times 10^{-7}$, and $c_{T O}=1.12$ in $\left(\mathrm{m}^{2} / \mathrm{s}\right) \times 10^{-7}$ and the coefficients $v_{L A}=9.01 \times 10^{3}, v_{T A}=5.23 \times 10^{3}$, $v_{L O}=0.00$ and $v_{T O}=-2.57 \times 10^{3} \mathrm{in} \mathrm{m} / \mathrm{s}$, is applied. Further, the value of $\omega_{0, b}$ is $0.00,0.00,9.88 \times 10^{13}$, and $10.2 \times 10^{13}$ in $\mathrm{rad} / \mathrm{s}$, respectively, for the branches $L A, T A, L O$, and $T O[41]$.

\subsection{Phonon drift}

After establishing the initialized phonons, they are allowed to move from one point to another. During this transport, the phonons may experience impacts with other phonons, or scattering from boundaries. In MC technique, the drift and scattering events are treated independently and subsequently. From mathematical point of view, this means that the transport and scattering parts of the BTE are discontiguous. In other words the BTE is decoupled in each time step into two equations: the collisionless equation, eq. 10, and the spatially homogeneous Boltzmann equation, eq. 11. The solution of the eq. 10 is used as the initial value for the second step where the eq. 11 is solved [42]. This operator 
separation introduces an error which is first-order in time.

$$
\frac{\partial f}{\partial t}+\mathbf{v}_{\mathbf{g}} \cdot \nabla \mathbf{f}=\mathbf{0}
$$

$$
\frac{\partial f}{\partial t}=\left[\frac{\partial f}{\partial t}\right]_{\text {scatter }}=\frac{f-f_{0}}{\tau} .
$$

The position of the phonons that drift inside the 3-D silicon MOSFET can be traced via using an explicit first order time integration,

$$
\mathbf{r}(\mathbf{t}+\Delta \mathbf{t})=\mathbf{r}(\mathbf{t})+\mathbf{v}_{\mathbf{g}} \Delta \mathbf{t} .
$$

In the above relation, $\Delta t$ is the time step. As the phonons drift, their frequency range may move from one frequency interval to the other one.

Hence, according to the new position vector the new spatial bin to which the phonon belongs must be determined. So, the drift results in redistribution of energy (and temperature) in the computational domain [42]. At the end of each drift, the energy of all the phonon ensembles of each cell are summed, and consequently the energy of each spatial frequency interval per unit volume, $\tilde{U}_{\text {cell }}$, is calculated. Then, the pseudo-temperature $\tilde{T}$ of each cell, which has no physical meaning, but is introduced for the sake 
of simplifying the calculation of the scattering processes, can be calculated by using the Newton-Raphson inversion method,

$$
\begin{array}{r}
\tilde{U}_{c e l l} . W=\sum_{p} \sum_{i=1}^{N_{b}} \hbar \omega_{c, i}\left[\exp \left(\frac{\hbar \omega_{c, i}}{k_{B} \tilde{T}}\right)-\exp \left(\frac{\hbar \omega_{c, i}}{k_{B} \tilde{T}_{r e f}}\right)\right]^{-1} \\
\left.\frac{|\mathbf{K}|^{2}}{2 \pi^{2}} \frac{d|\mathbf{K}|}{d \omega}\right|_{\omega_{c, i}} \Delta \omega_{i} .
\end{array}
$$

Here, $\tilde{T}$ is calculated by considering the presence of thermodynamic equilibrium within each frequency interval. The phonon may also hit the boundaries of the system during the drift. In the next section, these collisions as an important part of heat transport modeling in nano-scale devices for determining the change in position and location, will be discussed .

\subsection{Interaction with the boundaries}

Considering the free flow, the phonons which are moving in the space may hit the boundaries. Hence, it is necessary to follow the trend of the phonons to find out the boundaries at which the collision has taken place. In this subsection, an algorithm is given to check the circumstance under which each phonon could possibly hit the boundaries. In Fig. 2, the schematic of the the phonon movement is presented. In this figure, $\mathrm{O}$ is the origin and the 


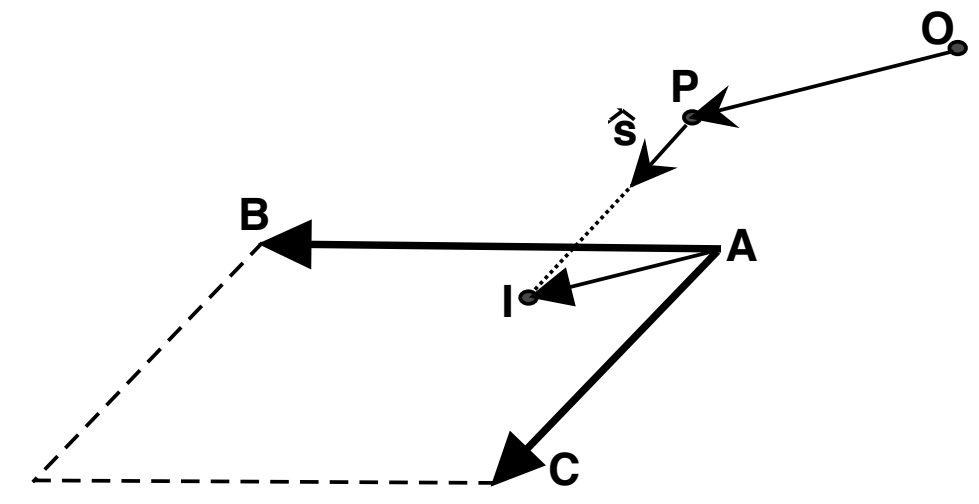

Figure 2: Phonon hitting the boundary in the three-dimensional space.

vector OP manifests the initial position of the phonon before the free drift. The desired phonon travels along the vector $\hat{s}$. Two sides of the following relation display the next position of the phonon, the vector OI $[43,40]$ :

$$
\mathbf{O I}=\mathbf{O P}+\epsilon \hat{\mathbf{s}}=\mathbf{O A}+\mu \mathbf{A B}+\nu \mathbf{A C} .
$$

If the conditions $0 \leq \mu, \nu \leq 1, \epsilon \geq 0$, and $\epsilon \leq v_{g} \times \Delta t$ are fulfilled, the point $I$ is placed in the boundary plane. The mentioned conditions should be checked for all boundaries of the computational domain. As the values of $\epsilon$ and $\mu$ are calculated, the point of the intersection of the phonon with the boundary is found as 


$$
\mathbf{O I}=\mathbf{O P}+\epsilon \hat{\mathbf{s}} .
$$

307 Next, the behaviour of the phonon ensemble after the collision should be

308

investigated. A phonon that hit the boundary can be reflected specularly or diffusively. In specular reflection the phonon with incident direction vector of $\hat{\mathbf{s}}_{\mathbf{i}}$ undergoes a mirror-like reflection. Hence, the direction vector of the reflected phonon, $\hat{\mathbf{s}}_{\mathbf{r}}$ will be

$$
\hat{\mathrm{s}}_{\mathrm{r}}=\hat{\mathrm{s}}_{\mathbf{i}}+2\left|\hat{\mathrm{s}}_{\mathrm{i}} \cdot \hat{\mathbf{n}}\right| \hat{\mathbf{n}} .
$$

Here, $\hat{\mathbf{n}}$ is the direction of the outward normal vector of the boundary. In real boundaries, the reflections are partially specular, and are distinguished by the value of specularity, $\alpha$. This factor has the value between zero (for completely diffusive) and one (for completely specular) reflections.

As a phonon ensemble hits a reflective boundary, a random number is taken into account. If the value of the random number is less than the value of specularity, the phonon is reflected specularly, otherwise, the reflection is diffusive. When the phonon undergoes the diffusive reflection, its velocity 
direction should be re-sampled as

$$
\hat{s}=\sin \theta \cos \phi \hat{x}+\sin \theta \sin \phi \hat{y}+\cos \theta \hat{z} .
$$

321 322

The degree of specularity varies for the 2-D and 3-D silicon materials. This is due to the difference between the number of diffusive boundaries of these cases. In the present paper, the dimension of the silicon MOSFET is $100 \mathrm{~nm}, 1 \mu \mathrm{m}$, and $50 \mathrm{~nm}$ in $X, Y$, and $Z$ directions, respectively. Hence, the value of specularity is taken to be equal to $0.885,0.98$, and 0.2228 , subsequently, for lateral planes parallel to the $X Y, X Z$, and $Y Z$ planes such that the predicted value of thermal conductivity matches the experimental data.

\subsection{Intrinsic Scattering}

A Phonon which travels in a solid can be scattered by means of lattice imperfections (i.e., vacancies, dislocations, and impurities), interactions with electrons, interactions with other phonons (intrinsic scattering), and boundaries. Each of these scattering, which itself can be elastic (means both energy and momentum are conserved) or inelastic (only energy is conserved), result in exchanging energy between different lattice wave vectors. 
Here, as the 3-D silicon MOSFET is assumed to be pure and perfect, the scattering due to the vacancies, dislocations and impurities is neglected. Three-phonon (Umklapp and normal) scattering, and scattering from boundaries are the mechanisms, which are considered in the present Monte-Carlo study of the silicon transistors.

Phonon-phonon scattering is the most important mechanism in the usual temperature range of interest $(300-600 \mathrm{~K})$ for a silicon MOSFET. Threephonon processes can be a normal $(N)$ processes conserving both energy and momentum or an Umklapp $(U)$ processes, which preserves the energy, but only conserves the momentum up to a reciprocal lattice vector. Hence, $U$ processes are the cause for the thermal resistance, while the $N$ processes cannot be neglected. At low temperatures, they involve in creation of sufficient number of high-K phonons that participate in U scattering. Therefore, it is essential to consider the contribution of both $\mathrm{N}$ and $\mathrm{U}$ processes for the calculation of the overall relaxation time, scattering rate. Here, like the previous works, the three phonon interactions are treated through a scattering rate [17].

$$
\tau_{N U}^{-1}=B_{L} \omega^{2} T^{3}(L A, \text { Normal }+ \text { Umklapp })
$$


353

and

$$
\tau_{N U}^{-1}=B_{T N} \omega T^{4}(\text { TA, Normal }) .
$$

354

355

$B_{L}$ and $B_{T N}$ are $2 \times 10^{-24}$ and $9.3 \times 10^{-13} \mathrm{deg}^{-3}$.

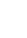

Narumanchi suggestion [19] in absence of the optical phonons as

$$
\begin{array}{r}
\frac{1}{\tau_{i, j}} \approx \frac{\chi \gamma^{2}}{3 \pi \rho v_{p h}^{2}\left|\mathbf{v}_{\mathbf{g}}\right|} \omega_{i} \omega_{t r} \omega_{j} r_{c}^{2} \mid\left[\frac{1}{\exp \left(\hbar \omega_{t r} / k_{B} T\right)-1}\right. \\
\left.-\frac{1}{\exp \left(\hbar \omega_{j} / k_{B} T\right)-1}\right] \mid
\end{array}
$$

Here, $v_{p h}$ is the phase speed of the input phonon which is the ratio of the frequency to the wave-vector, and $v_{g}$ is the output phonon group velocity that is the derivative of the dispersion relation relative to the wave-number. Also, $\omega_{j}$ is equal to $\omega_{i}+\omega_{t r}$, and the Grüneissen parameter, $\gamma_{b}$, which presents the strength of the scattering, is taken to be 0.59. $\chi$ is the degeneracy of the state at the boundary of the FBZ. In the equation above, $\omega_{t r}=\omega_{O}-\omega_{i}$, and $\omega_{O}$ is the optical phonon frequency. At last, the value of $r_{c}$ is calculated as [19]

$$
r_{c}=\frac{2 \pi / a-\left|\mathbf{K}_{\mathbf{i}}\right|}{2 \sqrt{2}} .
$$


Further, for the mechanism that the optical phonons are also taken part the scattering rate is calculated as

$$
\begin{array}{r}
\frac{1}{\tau_{i, j}} \approx \frac{\chi \gamma^{2}}{3 \pi \rho v_{p h}^{2}\left|\mathbf{v}_{\mathbf{g}}\right|} \omega_{i} \omega_{O} \omega_{t r}^{3}\left[\frac{1}{\exp \left(\hbar \omega_{t r} / k_{B} T\right)-1}\right. \\
\left.-\frac{1}{\exp \left(\hbar \omega_{O} / k_{B} T\right)-1}\right] .
\end{array}
$$

In this paper, the plots introduced by Henry and Chen for $L O$ and $T O$ branches are used to calculate the relaxation times of the optical phonons [44]. The curves are fitted to the mentioned plots for different temperatures in order to obtain the desired information. It should also be mentioned that the phonon-phonon scattering with the presence of four or more phonons are not taken into account. This is due to the fact that this type of scattering gets important at temperatures much higher than the Debye temperature which is far from the operating point of most nano-electronic devices. The obtained results for the relaxation times in different temperatures are given in Appendix 8.

In the Monte-Carlo simulation, the probability of scattering a phonons 
with the scattering rate of $\tau_{b}$ between time $t$ and $t+\Delta t$ is calculated as,

$$
P_{\text {scat }}=1-\exp \left(\frac{-\Delta t}{\tau}\right)
$$

Then, a random number $R_{\text {scat }}$ is chosen to determine weather the phonon has been actually scattered. If the probability of scattering, $P_{\text {scat }}$, is greater than $R_{\text {scat }}$, then the scattering is occurred. When a phonon scatters, its frequency, branch and direction will be re-sampled from the cumulative density function. Here, the time step, $\Delta t$ is chosen to be smaller than the minimum scattering rate of the phonons, which are sampled during the simulation. Consequently, as the scattering rates are temperature-dependent, the timestep also relies on temperature. To make sure that the rate of formation of phonons of a certain state is equal to its rate of destruction, the distribution function after scattering, $F_{\text {scat }}$, has to be modified by the probability of scattering:

$$
F_{\text {scat }}(\tilde{T})=\frac{\sum_{j=1}^{i} N_{j}(\tilde{T}) \times P_{\text {scat }, j}}{\sum_{j=1}^{N_{b}} N_{j}(\tilde{T}) \times P_{\text {scat }, j}}
$$

As the scattering reestablishes the equilibrium, the outgoing phonon states are re-sampled from an equilibrium distribution. By using $F_{\text {scat }}$ to re-sample phonon frequency, the energy is kept to be constant during the 
MC simulation. The rigorous implementation of momentum conservation is, however, harder to address. Since the MC process treats phonons one by one, $N$ or $U$ interactions cannot be rigorously treated.

There is also a challenge in re-sampling the phonons after scattering. Only Umklapp processes take part into the thermal resistance. On the other hand, the MC method treats phonons one by one. As changing the direction of the phonons is involved with the form of scattering, so only the direction of the phonons which scatter through a $U$ scattering, are randomly re-initialized. In this study, similar to the work by Lacroix et al [26], half of the scattered phonons are re-sampled for their directions. After modeling the scattering process, the energy within each spatial frequency bin is summed up, and local equilibrium temperature is calculated for each spatial bin. Then it is proceeded to the next time step.

\subsection{Heat generation modeling}

In the cube of $10 \times 10 \times 1000 \mathrm{~nm}^{3}$, located in the center of the silicon MOSFET, the heat is generated due to the electric current transport. Hence, this heat generation should be included in the Boltzmann equation. This term is modeled as a resource which heats the region by releasing the phonons into it. In order to prescribe the frequency to the released phonons, the 
413

temperature in which the phonons frequency are belonged is determined

$$
\begin{gathered}
\sum_{P} \sum_{i=1}^{N_{P}} \hbar \omega_{i}\left(f_{0}\left(\omega_{i}, T_{\text {pseudo }}\right)-f_{0}\left(\omega_{i}, T_{\text {ref }}\right)\right) D\left(\omega_{i}, P\right) \Delta \omega_{i}= \\
\frac{U_{\text {cell }} \times W}{\Delta X \Delta Y \Delta z}+\frac{E_{\text {gen }}}{\Delta X \Delta Y \Delta z} .
\end{gathered}
$$

414

$$
\sum_{i=1}^{N_{g e n}} \hbar \omega_{i} W \approx E_{g e n}(X, Y, Z)
$$

\section{Numerical considerations}

The written code has become parallel using the OpenMP, the time of calculation has notably decreased. While considering the parallel code, the problem is encountered in the generation of random numbers. This is due to the dependency of producing random numbers on one core. On the other side, in paralleling the code with OpenMP the memory is simultaneously 
used by the parallel processors. Since, each random number uses the previous random number as a core, the processors can not generate random numbers in parallel. Hence, the use of random number generation algorithm in parallel codes, not only increases the time of computation, but also makes the results wrong. Consequently, the new algorithm for introducing random numbers is written, in which, all the random numbers are created out of the parallel environment, and then are given to the parallel framework. Although the time consuming strategies have been taken into account, but, the MonteCarlo method is still too much time consuming, and accordingly needs high performance computers. To be more precise, 8 cores with the specifications of $2.8 G H z$ and $32 G B-R A M$ were put into service.

\section{Verification}

In the following sections, the obtained results of the Monte-Carlo simulation are presented. First, the success of the verification is reported. Then, the verification of computation is done for a simple cuboid in different conditions and the 2-D silicon MOSFET. 


\subsection{A cuboid in different regimes}

Conductive heat transfer is carried out in ballistic and diffusive regimes. In this section, the accuracy of the written code is checked for the situations in which one of the mechanisms is dominant in heat conduction through a cuboid.

When the temperature is very low and the environment is too small, the heat conduction is undertaken by the ballistic movement. In this limit, the temperature profile is obtained using the Stefan-Boltzmann law. According to this relation, the temperature all around the cuboid body is constant and is equal to $T_{\text {ballistic }}=\left[\frac{T_{h}^{4}+T_{c}^{4}}{2}\right]^{\frac{1}{4}}$. For a cuboid of size $L_{x}=4 \times 10^{-7} \mathrm{~m}$ and $L_{y}=L_{z}=5 \times 10^{-7} \mathrm{~m}$, the temperature of the cold and hot walls are taken to be $3 \mathrm{~K}$ and $11.88 \mathrm{~K}$, respectively. As a consequence, the temperature of the body should be $10 \mathrm{~K}$. Besides, as the heat transfer happens along the $X$ direction, this direction is divided into 40 segments. Fig. 3 (a) displays that the results of the written code and the precise theoretic solution fit almost very well to each other.

The next sample is the one, in which, the diffusive transport is the predominant heat conduction mechanism. This situation is achieved at high temperatures and large sizes. In this limit which is the high Knudsen num- 
ber regime, the temperature distribution is attained using the Fourier's law. Due to the model, the temperature varies linearly at steady state condition. The specimen is again a cuboid, but with size of $L_{x}=4 \times 10^{-6} \mathrm{~m}$ and $L_{y}=L_{z}=5 \times 10^{-7} \mathrm{~m}$. The temperature of the hotter boundary is $310 \mathrm{~K}$, while the cold boundary is kept at $290 \mathrm{~K}$. The Fig. 3 (b), demonstrates the acquired behaviour of the temperature profile from the transient regime till it reaches the steady state in $X$ direction. It is obvious that the linear behaviour of the steady state assures the correctness of the calculations.

\subsection{A cuboid with uniform heat source}

Another system of interest is the one involving the heat source term. As mentioned in section 3.7, the presence of this term in the Monte-Carlo calculation is declared by injecting phonons into the computational domain such that the generated energy of the source gets equal to the energy of these phonons. In this section, the accuracy of this assumption is checked for a simple cube of $L_{x}=4 \times 10^{-6} \mathrm{~m}$ and $L_{y}=L_{z}=5 \times 10^{-7} \mathrm{~m}$. The heat generation is monotonous and equal to $\ddot{g}=2 \times 10^{15} \frac{\mathrm{W}}{\mathrm{m}^{3}}$. The temperature profile is obtained using both the precise solution and the Monte-Carlo simulation. The 


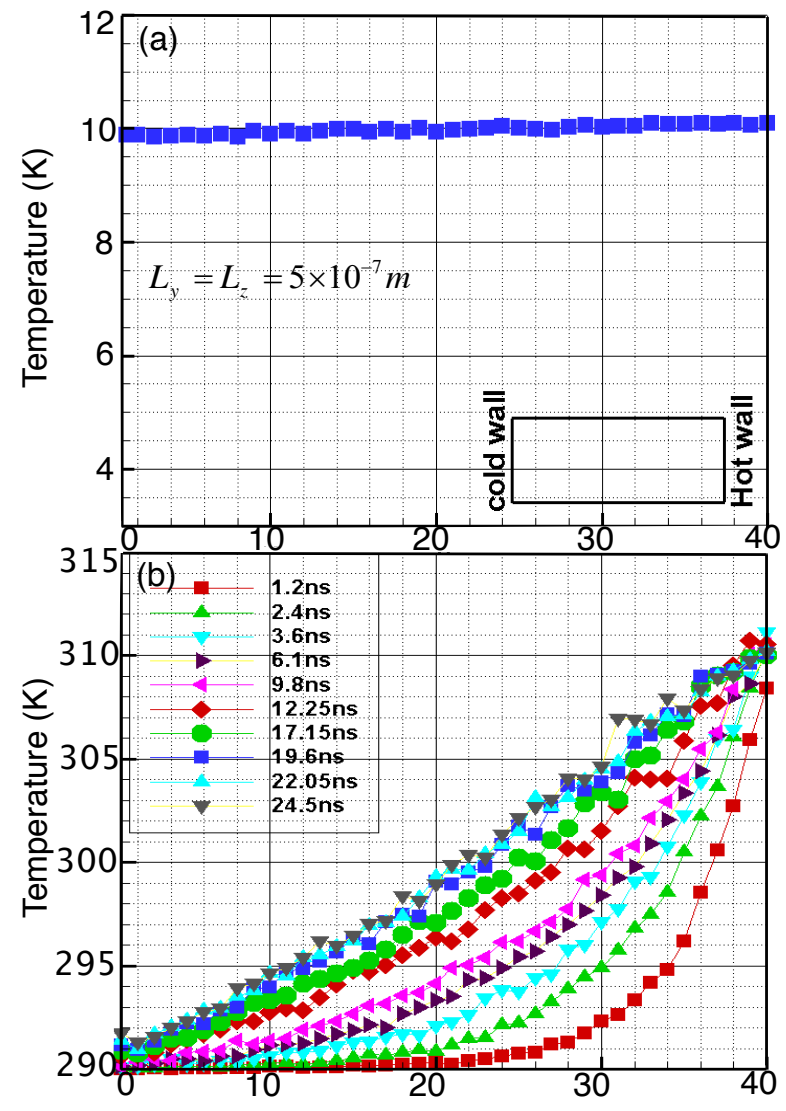

Figure 3: (a) The solution in ballistic movement limit along $X$ direction. (b) The transient regime in diffusive limit at $X$ direction. 
477

theoretical solution is found via solving the following Fourier-based equation:

$$
\nabla \cdot\left(K_{T} \nabla T\right)+\ddot{g}^{m}=0 ; \quad K_{T}=\frac{1.585 \times 10^{5}}{T^{1.23}}
$$

478 479

which results in a temperature profile of subsequent form along the $X$ direction,

$$
T(x)=\frac{1}{\left(-6834.45 \times x+\frac{4.6 \times 10^{11}}{317} x^{2}+2.71 \times 10^{-11}\right)^{\left(\frac{100}{23}\right)}}
$$

This solution and the other one calculated from the Monte-Carlo simulation of the Boltzmann equation are shown in Fig. 4. Due to the statistical nature of the Monte-Carlo simulation, some noises are appeared in the solution. Although, the reduction of $W$ makes the fluctuation to decrease, but because of the increased computational cost it is not recommended. Another method to obtain the more precise results in steady state is summing up the temperatures acquired from different times and calculating the time average. This method has computational advantageous, and enhances the reliability of the results. 


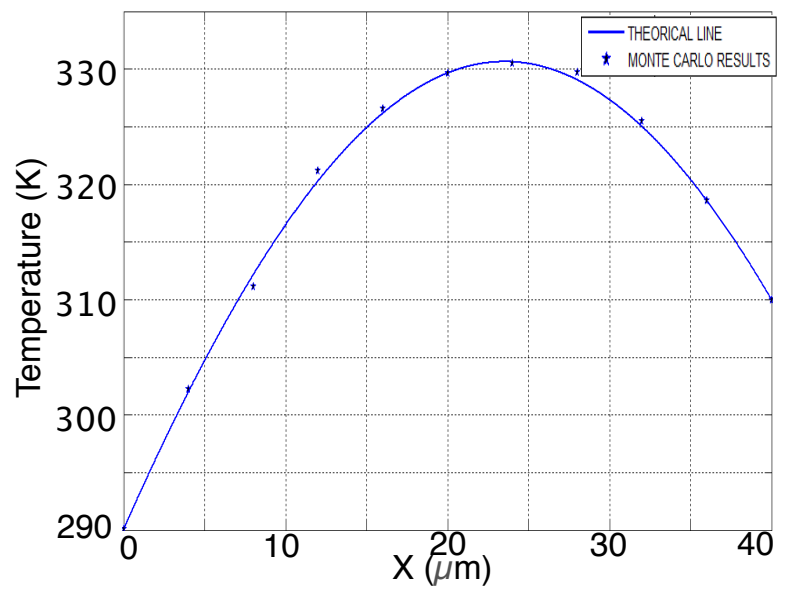

Figure 4: The temperature profile for a cuboid with the heat generation region.

\subsection{2-D silicon MOSFET}

To make sure about the accuracy of the written 3-D Monte-Carlo code, the calculations are carried out for the 2-D silicon MOSFET. The results obtained for case I, are compared to those of Refs. [16] and [45]. In similarity to the work done by Pop et al. [45], the heat generation zone of $1 \times 10^{19}\left(\mathrm{~W} / \mathrm{m}^{3}\right)$ at the middle top of the 2-D MOSFET is on for the first $10 \mathrm{ps}$. Fig. 5, presents the comparison between the peak temperature rise versus time obtained from the present code and the available data [45] during starting $10 \mathrm{ps}$.

As it is seen, there is a good consistency between the achieved results and those of the previous studies. This reveals the accuracy of the written code and makes it a reliable calculation. Throughout the time of switching 


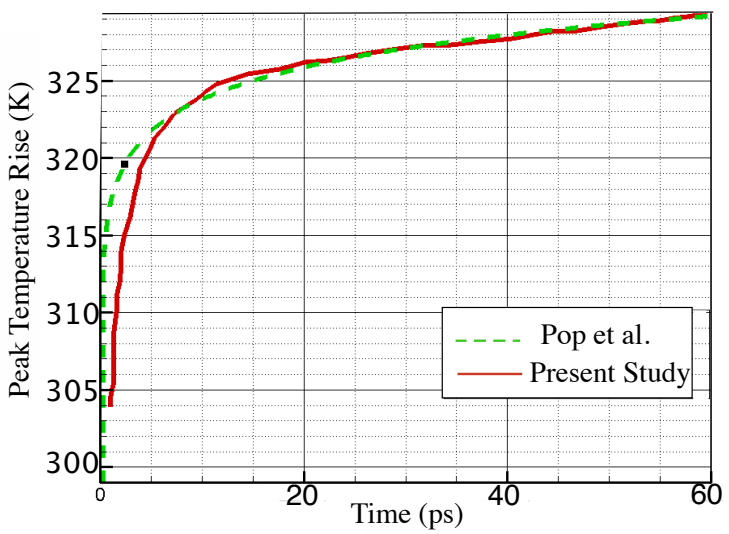

Figure 5: The peak temperature rise relative to time for a 2-D silicon MOSFET transistors.

on the heat generation zone, the temperature increases very fast. Figure 6(a) manifests that turning off the heat source, the MOSFET does not immediately starts to get cool, and the maximum temperature occurs at $t=13 \mathrm{ps}$. The temperature enhancement after switching off the heat generation zone, indicates the presence of non-equilibrium heat transfer. In other words, the accumulation of phonons in a limited region, makes them to be pumped out with delay. The ballistic movement causes the phonons to diffuse in all directions and such diffusion results in the reduction of heat reaching the heat exchange boundaries. The temperature contour at $t=60 \mathrm{ps}$, is manifested in Fig. 6(b). It is obtained that it takes $50 \mathrm{ps}$, five times greater than the heating time, for the 2-D MOSFET to get almost cool. This finding demonstrates deficiency in the design of the MOSFETs. 

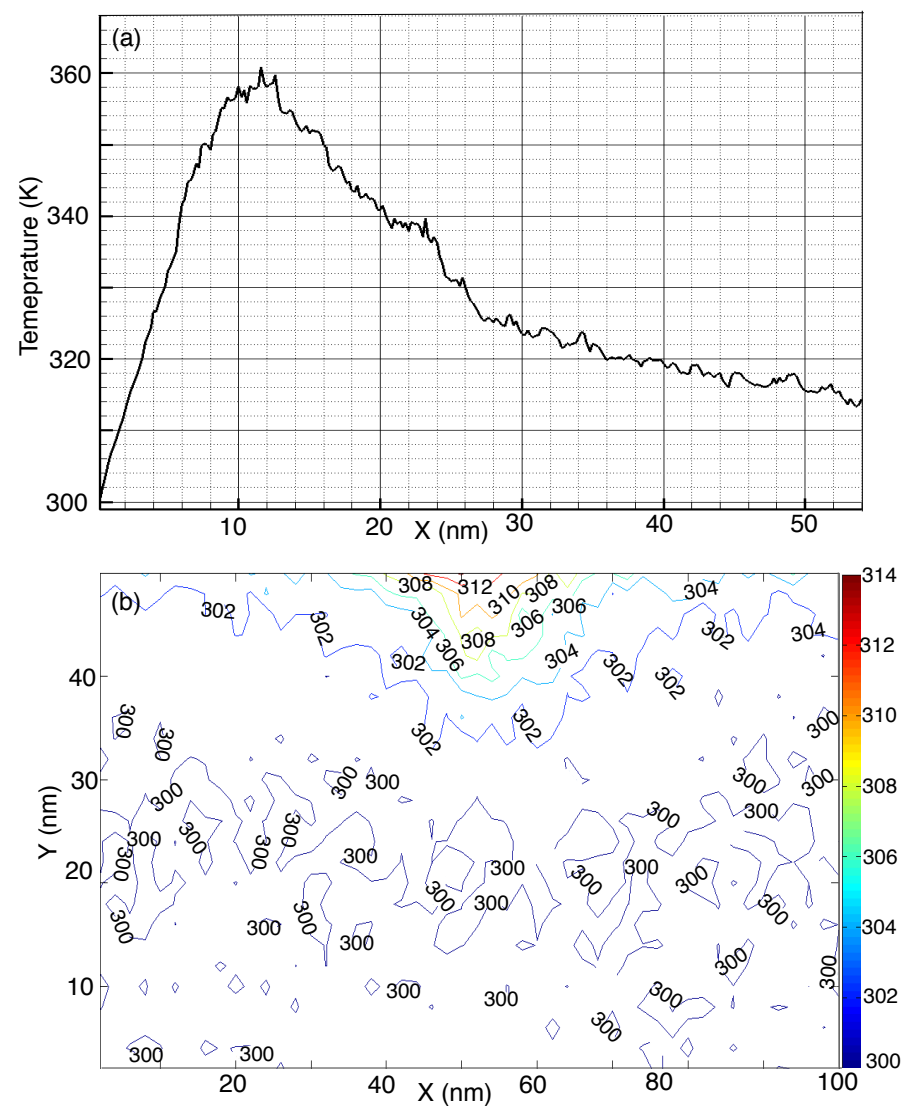

Figure 6: (a) The maximum temperature relative to the time for a 2-D MOSFET during heating-cooling procedure. (b) The temperature contours at $t=60 \mathrm{ps}$. 


\section{Results and Discussions}

As mentioned, the three-dimensional Monte-Carlo simulation is performed to study the heat transfer in two 3-D silicon MOSFETs with different boundary conditions. In case one the heat is only transferred within the bottom boundary, while the second case is also involved with heat emitting from two separate portions of the top boundary in addition to the energy transferred from the underneath. In addition, the heat generation is considered to be inhomogeneous in both transistors.

\subsection{Case one}

The case one is investigated in two distinct situations. First, the 3-D MOSFET is affected by the heat source during whole $500 \mathrm{ps}$ of computation time. The entire system is initially kept at $299 \mathrm{~K}$. The peak temperature versus time-step number is plotted in Fig. 7(a). As it is seen, the peak temperature starts to decrease after times greater than $200 \mathrm{ps}$. This finding can be justified by analyzing the input/output energy plots. In Fig. 7(b), the blue line presents the energy which is exited from the bottom boundary of the nano-device into the environment. The black line shows the whole input energy that is the summation of the gen- 
erated heat in the gate, violet line, and the income energy from the boundaries, the red line. In more details, a number of phonons as a source of heat generation and the input heat are entered to the computational space. Also, some phonons leave this space from the bottom boundary. After $t=200 \mathrm{ps}$, the trend of the plots reverses, and the output energy becomes larger than the input energy. Consequently, the temperature decrease between $44 \mathrm{ps}$ and $66 \mathrm{ps}$, is the result of heat egression from the open boundary. In other words, it takes $44 \mathrm{ps}$ for phonons to travel the $50 \mathrm{~nm}$ distance to touch the underneath boundary, and alter the temperature profile. Hence, the group velocity of this type of phonons is $1022.73 \frac{\mathrm{m}}{\mathrm{s}}$, which determines that they belong to the optical branches or the TA mode.

Further, it is obtained that around $t=22 \mathrm{ps}$, a temperature jump of $60 \mathrm{~K}$ occurs. This abrupt growth takes place when the peak temperature has reached the temperature $400 \mathrm{~K}$. The phonons that do not diffuse through the MOSFET, gather in a small region and make the hot spot. In the present 3-D transistor, the hot phonons are piled up in a zone with $50 \mathrm{~nm}<X<70 \mathrm{~nm}$ and $30 \mathrm{~nm}<Z<50 \mathrm{~nm}$. It is found that before the appearance of the temperature jump, the acoustic phonons are the prevailing phonons existed in the hot-spot. As a consequence, due to their larger group velocity relative 


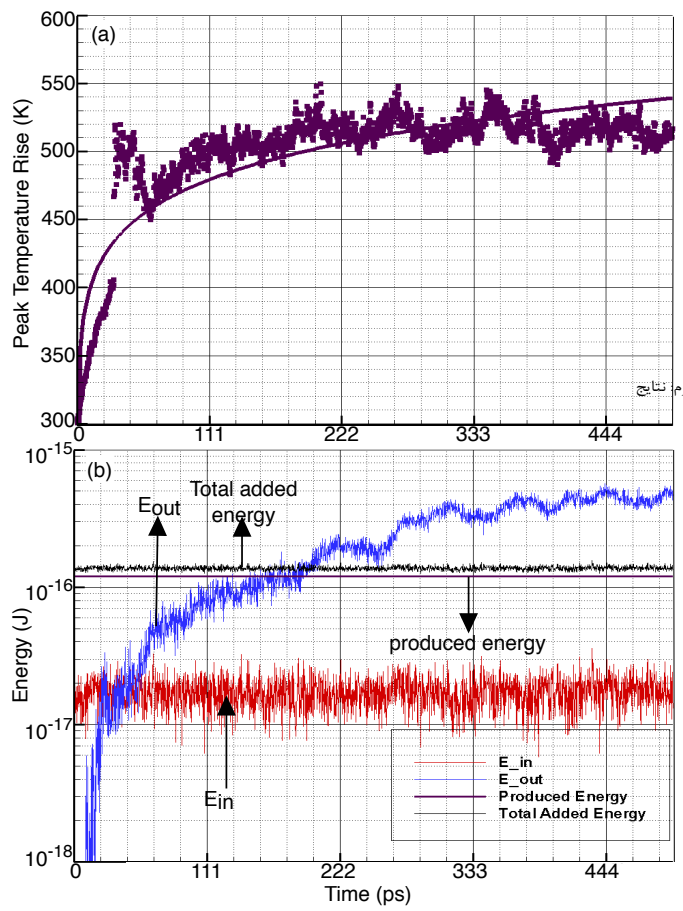

Figure 7: (a) The peak temperature rise versus time for a 3-D MOSFET in the presence of the heat zone. (b) The trend of energy in 3-D MOSFET boundaries. The blue line shows the energy exited from the bottom boundary of the nano-device into the environment. The black line shows the whole input energy that is the summation of the generated heat in the gate violet line, and the income energy from the boundaries, the red line. 
to the optical phonons, they carry away the heat from the hot-zone. On the other side, as time evolves and the jump happens, the number of acoustic phonons in the hot-spot region decreases while the amount of optical phonons grows. The group velocity of the optical phonons are lower then that of the acoustic phonons, consequently, in a specified period of time, the amount of the heat carried out of this region by these dominant phonons significantly decreases. Hence, the reduction of the heat dissipation arises, which causes this region to experience augmentation of heat energy. The transmutation in the phonon combination towards the optical phonons with lower group velocity, results in the occurrence of temperature-jump in the hot spot. Moreover, in a hotter zone, more phonons with higher frequencies are produced. This leads to the more increment of the temperature in this region.

In the following, the temperature profiles in various $X Z, Y Z$, and $X Y$ planes of the 3-D silicon structure are investigated. The temperature distribution in $X Z$ plane is manifested for four different times in Figs. 8(a-d). Figure 8 presents the profile for the time before the abrupt jump in the maximum temperature. As it is shown, more than half of the heat energy is stored in the hot-spot, which is created in the middle top of the MOSFET. 
This notable energy makes the temperature of this zone to increase.

The heat localization in the hot-spot is shown in Fig. 8(b). This figure reveals the temperature profile after the temperature skip. As it is seen from Fig. 8(c), the first thermal wave reaches the bottom open boundary at $t=49.5 \mathrm{ps}$. Therefore, the minimum temperature of the 3-D MOSFET, which belongs to the areas far from the hot-spot, gets larger. Further, Fig. 8(d) presents the temperature profile in $t=200 \mathrm{ps}$. At this time, the heat removal from the underneath boundary becomes more than the input energy. Then, the transistor starts to get cool, and the heat energy moves from the heat generation zone to the inner parts to make the MOSFET attain the equilibrium state. Finally, the steady-state temperature distribution is demonstrated in Fig. 8(e).

In addition, to analyze the 3-D heat transfer more precisely, the temperature distribution in other planes is also investigated at $t=500 \mathrm{ps}$. Figs. 9(a) and (b) display the temperature contours in $Y Z$ plane for two different values of $X=2$ and $60 \mathrm{~nm}$. Also, the deportment of temperature profiles along the $X Y$ plane in various $Z$, is shown in Figs. 9(c-e). As it is demonstrated in Figs. 9(a) and (b), due to the non-uniform generated heat, the heat flux spread in the places nearer to the top-left boundaries is remarkably less than 


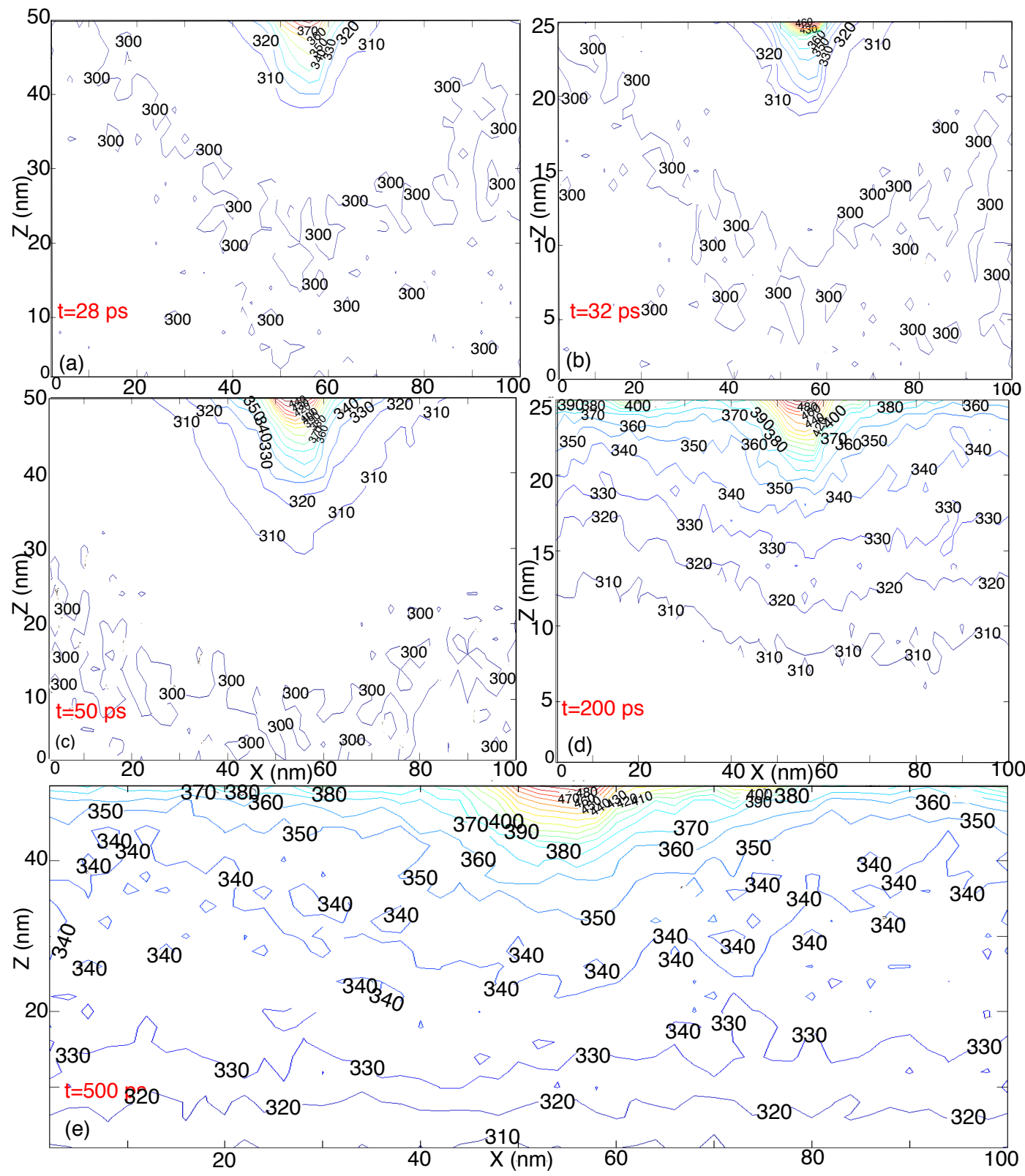

Figure 8: (a-e) The temperature distribution in $X Z$ plane averaged over $Y$ direction, respectively at $t=28,32,50,200$, and $500 \mathrm{ps}$. 
that of released through the right area. Besides, the penetration of the heat into the inner parts of the MOSFET and the increase in the temperature of the lower parts of the transistor is obvious. In Figs. 9 (c-e), the temperature profile in $X Y$ plane for the bottom, the lowest part of the heat generation zone, and the top of the MOSFET is displayed. The ballistic movement creates heat islands in cross sections of the transistors. These islands are more visible around the heat source owing to the presence of more phonons. Also, they are more likely to be formed in proximity of the left side of the MOSFET. This is for the reason that the lower temperature of the left side of the heat generation zone, causes the production of the phonons with less energy and larger velocity.

It is acquired that at $t=500 \mathrm{ps}$, the 16 percent of the energy which is mostly carried by $T O$ phonons, is still within the heat generation region. This shows that the equilibrium process has taken place, and the heat is propagated around the MOSFET to reduce the maximum temperature. Hence, the temperature augmentation occurs in the upper one fifth of the transistor while the remained four fifth of the MOSFET has lower temperature close to the initial temperature. Also, it has been presented in Figs. 8(a-d) that the generated heat is concentrated in a limited zone called as a hot-spot. This 


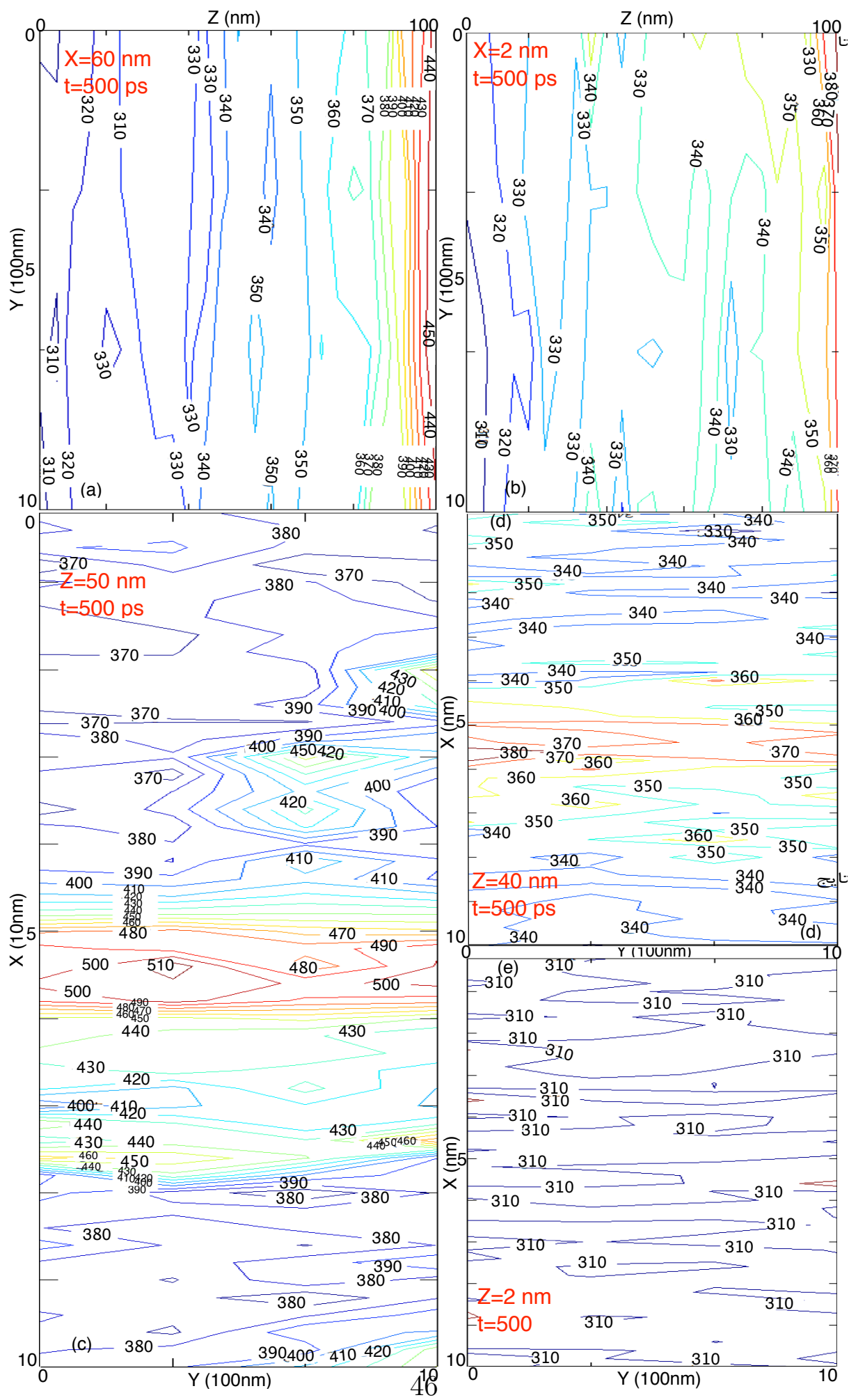

Figure 9: (a) The temperature profile for a 3-D MOSFET in the $Y Z$ plane at $X=60 \mathrm{~nm}$ and in $t=500 \mathrm{ps}$ in the presence of the heat zone. (b) The same as (a) but for $X=2 \mathrm{~nm}$. (c-e) The temperature behaviour in $X Y$ plane at $t=500 \mathrm{ps}$, respectively, for $Z=50,40$, and $2 \mathrm{~nm}$. 
deficiency makes the lifetime of the transistor to decrease. In this wrongly designed MOSFETs, the heat transfer firstly occurs in the $X$ direction, so the temperature as isotherm waves moves along the $Z$ direction. The heat transfer in the $X$ direction instead of the one in $Z$, causes the time for temperature enhancement to increase. The newly designed 3-D transistors have overcome this failure by dispreading the heat all over the MOSFET.

At the next step, the heat source of the mentioned 3-D silicon transistor is terminated at $t=500 \mathrm{ps}$, and the system is cooled by the energy exchange with the environment until $t=1 \mathrm{~ns}$. The computational time of this simulation with mentioned $\mathrm{CPU}$ characteristics was 2 days, 13 hours and 29 minutes. Figure 10, manifests the behaviour of the peak temperature after switching off the transistor, for the time period of $500 \mathrm{ps}$ or up to the time $t=1 \mathrm{~ns}$. It is obvious that for the first $220 \mathrm{ps}$, the temperature suffers many fluctuations until the expecting descending trend appears at $t=726 \mathrm{ps}$.

In the following, the behaviour of the temperature profile will be analyzed in more details. Just as the heat source is turned off, an abrupt temperature reduction becomes evident. This prompt reduction trend lasts for nearly $11 \mathrm{ps}$ and then the drastic fluctuations occur. Again, $60 \mathrm{ps}$ after the disconnection of the source/drain current, the temperature starts to grow 


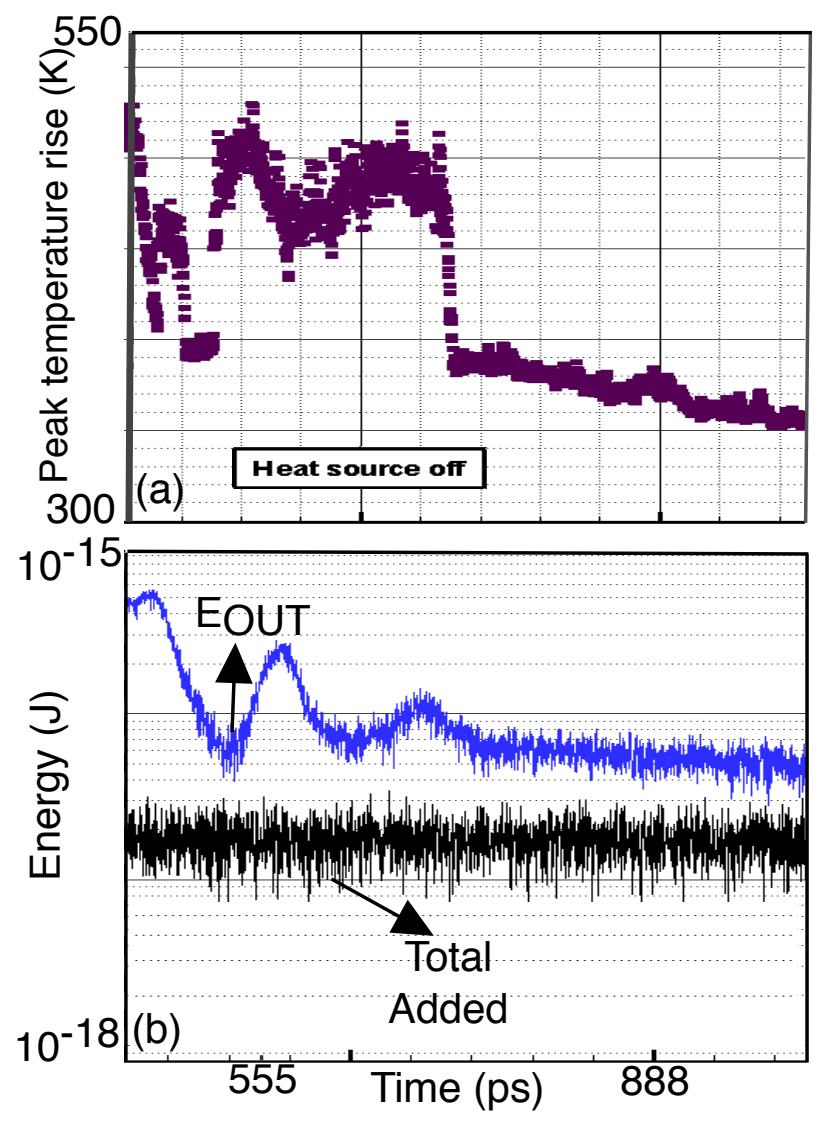

Figure 10: (a) The peak temperature rise versus time for a 3-D MOSFET when the heat source is turned off. (b) The energy behaviour in 3-D MOSFET boundaries. The blue line shows the energy exited from the bottom boundary of the nanodevice into the environment. The black line shows the whole input energy which in the cooling time is equal to the income energy from the boundaries. 
unexpectedly. Moreover a temperature jump takes place and then the temperature remains constant for an interval. Finally as previously remarked, at $t=236 \mathrm{ps}$ the temperature experiences the conceivably reductive trend.

Figure 10, also exhibits that 500 ps after switching off the transistor, the same as the time of heating, the maximum temperature in the MOSFET is $50 \mathrm{~K}$ higher than the temperature of the environment. The inability of the transistor to be cooled in the same time as the time spent for heating, along with the creation of the hot-spot are the two main difficulties of the 3-D MOSFETs.

In this part, the reason for the occurrence of such deportment is given. Two mechanisms are responsible for decreasing the peak temperature of the nano-device. The first procedure which is presented by the blue solid line in Fig. 10(b) is exiting the heat from the boundary. The other mechanism is the heat diffusion from the hot zone to the inner parts of the nano-system. The most important time interval is between $500 \mathrm{ps}$ and $744.144 \mathrm{ps}$ in which the MOSFET is affected under the current disconnection shock. The study of the disappearing of the hot spot and the transformation of the phonons from one branch to another kind of the branch gives accu- 
rate information about the process of cooling the transistor. The mechanism that firstly causes the intense temperature reduction in the device is the heat diffusion inside the device. Switching off the heat source, the abrupt temperature decrease presents itself because of the heat propagation in the transistor. During this mechanism the hot spot zone destroys while transferring the heat to the other areas of the nano-device. As a consequence of the presence of five boundaries around the center of the heat generation zone, the heat distribution along the $X$ direction is somehow imprisoned, and accordingly the heat transfer is more visible alongside in comparison to the heat diffused through the $Z$ direction. Although the temperature gradient along the $Z$ direction is almost constant, as it seen in Fig. 11(a), the heat diffusion across the $X$ direction makes the heat propagation from the hot zone the most effective temperature reducer for a time interval of $26 \mathrm{ps}$. After the time $t=526 \mathrm{ps}$, the heat is nearly distributed along the $X$-direction, and the heat transfer through the $Z$ direction decreases the maximum temperature. Also, the Fig. 11(b) demonstrates the poor heat propagation along $Z$ until the time $t=526 \mathrm{ps}$.

As the time passes, the two procedure of heat propagation from the hottest region and the heat exchange with the environment from the bot- 

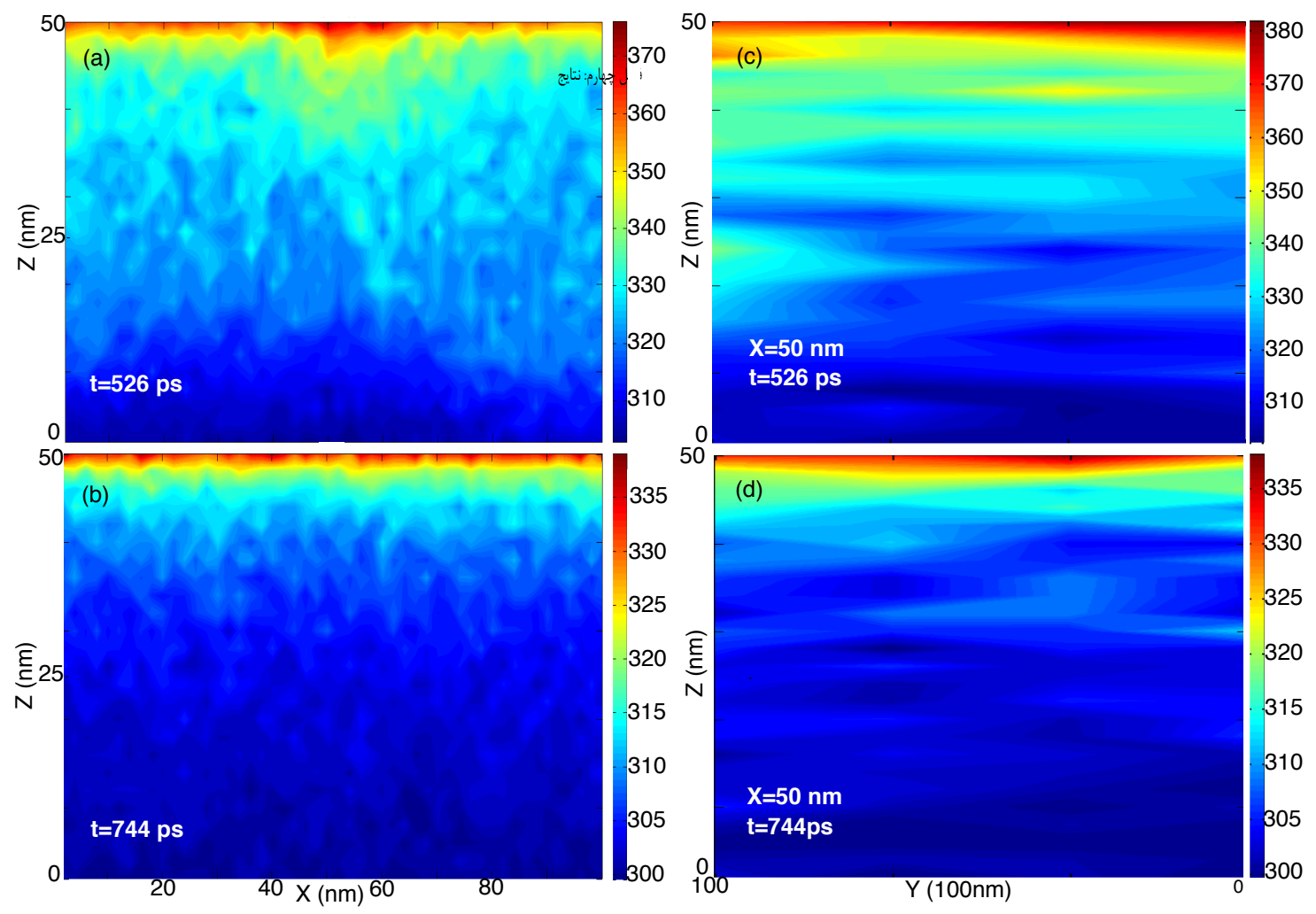

Figure 11: (a) The $Y$-averaged temperature distribution in the $X Z$ plane at $t=526 \mathrm{ps}$ when the heat zone is switched off at $t=500 \mathrm{ps}$. (b) The same as (a) but for $t=744 \mathrm{ps}$. (c) The temperature deportment in $Y Z$ plane at $X=50 \mathrm{~nm}$ when $t=526 \mathrm{ps}$. (d) Alike the (c) but when $t=744 \mathrm{ps}$. 


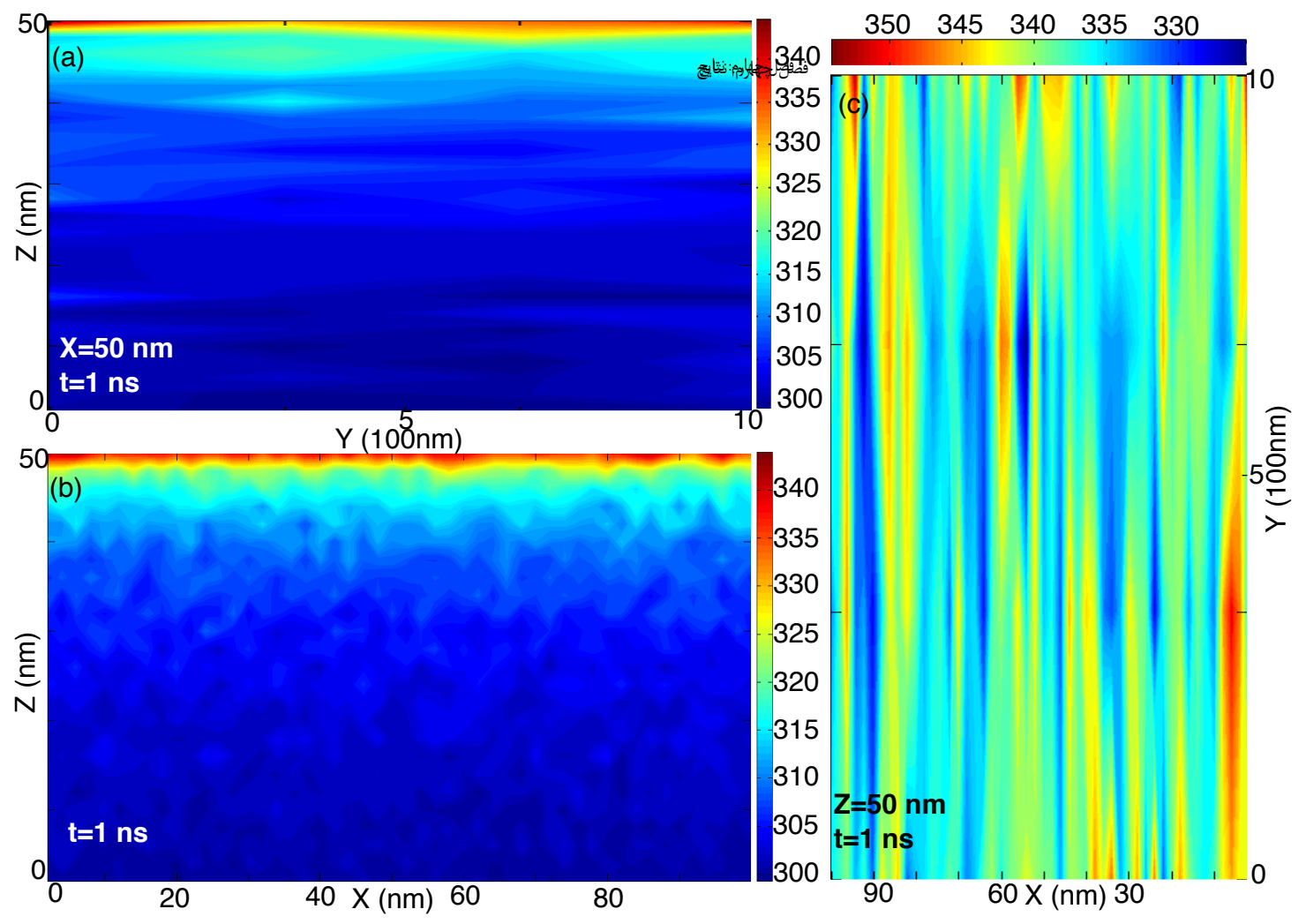

Figure 12: (a) The temperature profile for a 3-D MOSFET in the $Y Z$ plane at $X=50 \mathrm{~nm}$ when $t=1 \mathrm{~ns}$. (b) The temperature distribution averaged over $Y$ direction in the $X Z$ plane at $t=1 \mathrm{~ns}$. (c) The temperature behaviour over the $X Y$ plane at $Z=50 \mathrm{~nm}$ and $t=1 n s$. 
tom boundary cause the temperature all over the 3-D transistor to lessen. nevertheless, as Fig. 11(c) displays at $t=744 \mathrm{ps}$, the limited heat energy is confined on top of the 3-D MOSFET, and does not evacuate to the other places. This heat accumulation in a narrow area on top of transistor, dispenses the critical deficiency in transistor design. Also as the Fig. 11(d) suggests the heat propagation along the $Z$-direction have become faster at $t=744 \mathrm{ps}$. It is also obtained that the type of the phonon branch is a significant parameter in heat transfer behaviour of device. In the cooling process of the transistor, the type of the dominant phonons changes from the optical branch to the acoustic one. Consequently, the group velocity of the phonons increases, and the ballistic behaviour of the heat transfer gets stronger. As mentioned, first, the heat diffuses from the center of the hot-spot to all around the MOSFET, but then the heat energy in upper parts of the transistor imprisons between the five boundaries. Figures. 12(a-c) manifest the temperature profiles in $X Z, Y Z$, and $X Y$ planes 500 ps after switching off the heat source. In order to rectify the deficiencies of the 3-D transistor, the second case with improved boundary conditions is investigated. 


\subsection{Case two}

In order to resolve the deficiencies appeared in the case one, the transistor of case 2 is introduced. In this type of transistor, in addition to the heat exchange from the bottom boundary, the open boundary is considered in both sides of the top boundary. The size of the new designed MOSFET is the same as that of the case one. The heat generation is also non-uniformly distributed.

At first, the heat source is switched on for the whole $500 \mathrm{ps}$ time of the simulation. Figure 13(a) presents the peak temperature versus time. Also, the power curve fitted to the obtained data is displayed on Fig. 13(a). The $W$ factor is considered to be 184 . The Fig. 13(b) manifests the behaviour of the energy current at the boundaries. As it is seen, the output heat energy is equal to the input energy between the time interval of $t=22.2 \mathrm{ps}$ to $t=44.4 \mathrm{ps}$. Hence, the maximum temperature remains constant during this period of time. After this time, due to the more generated heat relative to the lost energy, the peak temperature arises until $t=88.8 \mathrm{ps}$. Then, ignoring the fluctuations occurred because of the statistical behaviour of the numerical method, and the nature of the ballistic movement, the maximum temperature follows the reduction trend. 


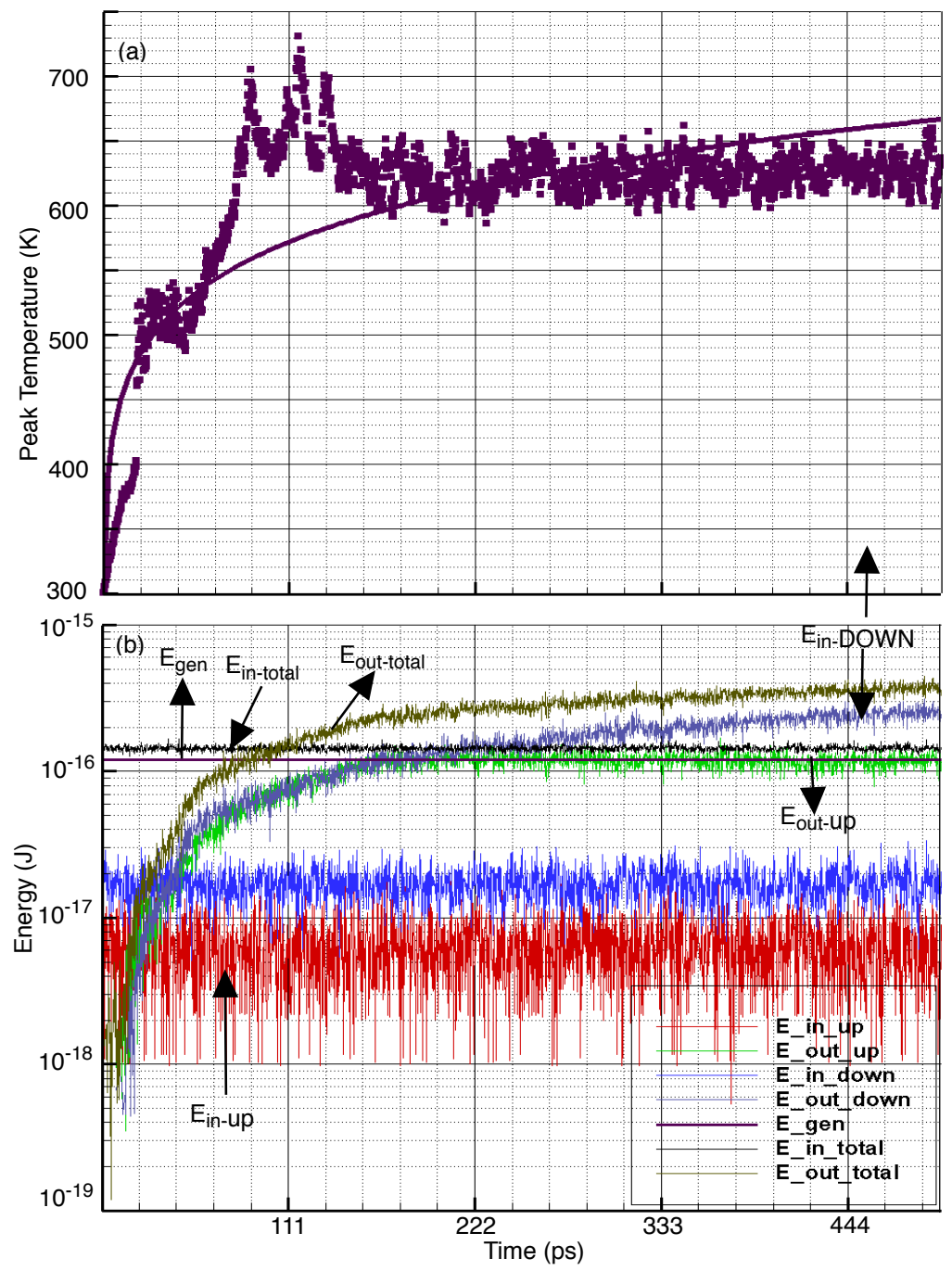

Figure 13: (a) The peak temperature rise versus time for a 3-D MOSFET with two top open boundaries and the open bottom boundary through the heating. (b) The energy behaviour of the 3-D MOSFET with new defined advantageous boundary conditions. 
Reaching the time of $t=222.2 \mathrm{ps}$, the heat pumped to the environment from the top open boundaries and the heat exited from the bottom boundary get commensurate. As described the area of the top open boundaries are likely one fifth of that of the bottom boundary. This fact reveals that the temperature gradient on top of the MOSFET is notably larger than the temperature slope in the bottom boundary. The temperature profiles in $Y Z$ planes, which are averaged along the $X$ direction for two intervals of $0<X<10 \mathrm{~nm}$ and $90<X<100 \mathrm{~nm}$ are respectively manifested in Figs. 14 (a) and (b). This averaging is performed through the places of the top open boundaries at $t=200 \mathrm{ps}$. This figures verify the larger gradient near the top boundaries specially at the left one. Also, the temperature distributions on the same positions for $t=500$ ps are plotted in Figs. 15(a) and (b). It is clear that the temperature gradient at the bottom boundary has been increased at $t=500 \mathrm{ps}$. Hence, the exited heat from the underneath border becomes larger than the energy sent out from the top open boundaries.

The comparison of the plot demonstrated in the Fig. 7(a) with that of Fig. 13(a), reveals that the presence of two open boundaries on top of the 3-D MOSFET strengthens the effect of hot-spot formation. To be more precise, the value of the maximum temperature of the second case increases more 

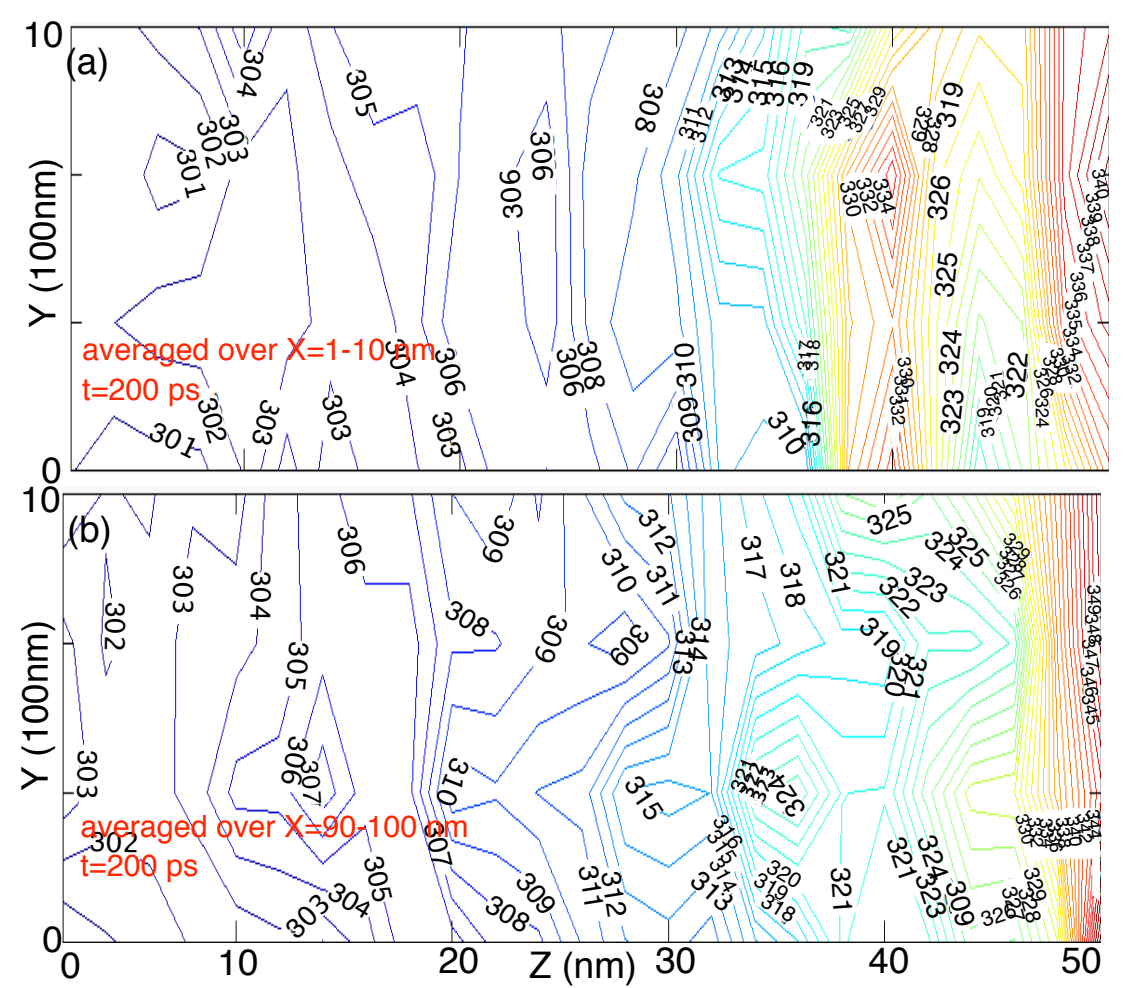

Figure 14: (a) The temperature profile in $Y Z$ plane averaged over the $X=1$ to $10 \mathrm{~nm}$ top open boundary when $t=200 \mathrm{ps}$. (b) The temperature distribution across the $Y Z$ plane averaged along the $X=90$ to $100 \mathrm{~nm}$ top open boundary. 


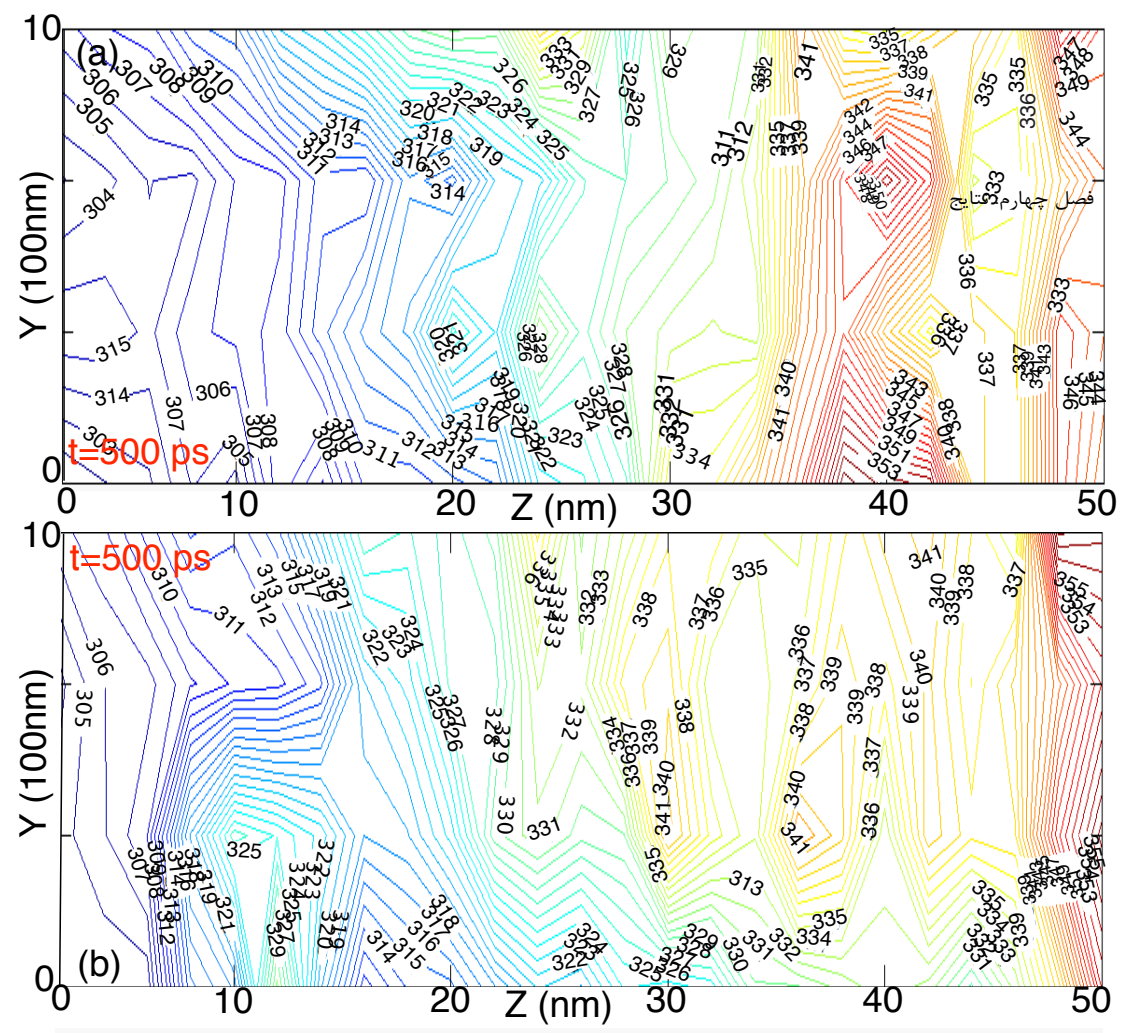

(c)

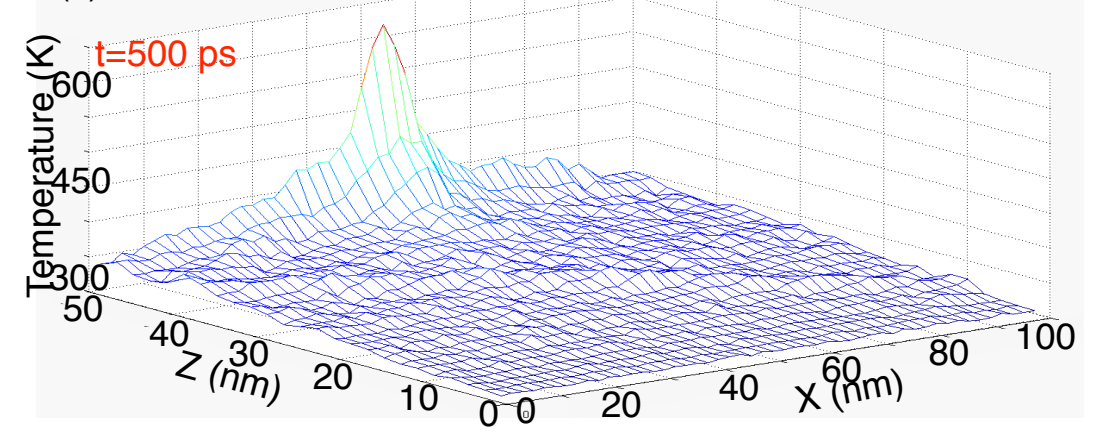

Figure 15: (a,b) The same as Fig. 13(a,b) but when $t=500$ ps. (c) The 3D plot of $Y$-averaged temperature profile in $X Z$ plane at $t=500 \mathrm{ps}$. 
and faster then the peak temperature appeared in the first geometry. Also, Figs. 13(b) and 7 (b) manifest that although the input energy of the both geometries are the same, the total output energy of the second case grows further and more rapid. The enhancement of hot-spot influence is due to the reduction of the temperature gradient along the $Z$-direction which itself is the result of the presence of the two open top boundaries. In spite of the fact that the total output heat is larger for case 2 , the less gradient makes the energy to concentrate more in a hot-spot, and inevitably less heat reaches the bottom boundary in a longer time.

It is obtained that the time effect is more pronounced than the size influence for the second geometry. In other words, the heat concentration in the MOSFET is due to the short time of the heat exchange, and accordingly the important procedure in the heat removal is the heat diffusion in the MOSFET rather than the heat exchange with the surroundings. It should be added that the amount of heat energy which is trapped in the hot-spot is $17.7 \%$ of the total energy for the second case, while this percentage for the first geometry is $15.9 \%$. But, as the total energy of the case two is lower than the case one, the heat energy inside the first geometry is lower. In addition, the heat concentration in the hot-spot can be attributed to the presence of 
less TA phonons due to more scattering.

After $t=500 \mathrm{ps}$, similar to case 1 , the heat source is switched off. Then, the thermal cooling mechanism is studied for the next $500 \mathrm{ps}$. The $W$ factor is taken to be 184 . The simulation has been performed for 2 days, 9 hours, 17 minutes and 31 seconds to get the result. The obtained peak temperaturerise and the energy current are displayed in Figs. 16 (a) and (b). The comparison between Fig. 16 (a) and Fig. 10 (a) reveals that although the top open boundaries cause more temperature enhancement throughout the heating, but they make the cooling simpler. Once the heat generation is stopped, more heat is transferred through the top boundary which has a larger temperature gradient, and consequently the heat does not accumulate on top of the MOSFET.

Fig. 17(a) shows the temperature distribution which is averaged over the $Y$-direction at $t=600 \mathrm{ps}$. It is seen that the maximum temperature which is $644.86 \mathrm{~K}$ at the time when the heat source is turned off, reduces to $387.21 \mathrm{~K}$. This shows $40 \%$ lessening of the peak temperature when $100 \mathrm{ps}$ has been passed. Also, the acquired temperature profile in $Y Z$ plan at $X=50 \mathrm{~nm}$ is shown in Fig. 17(b). This figure indicates that the effect of the bottom boundary in heat removal is not significant. Moreover, the behaviour of the 

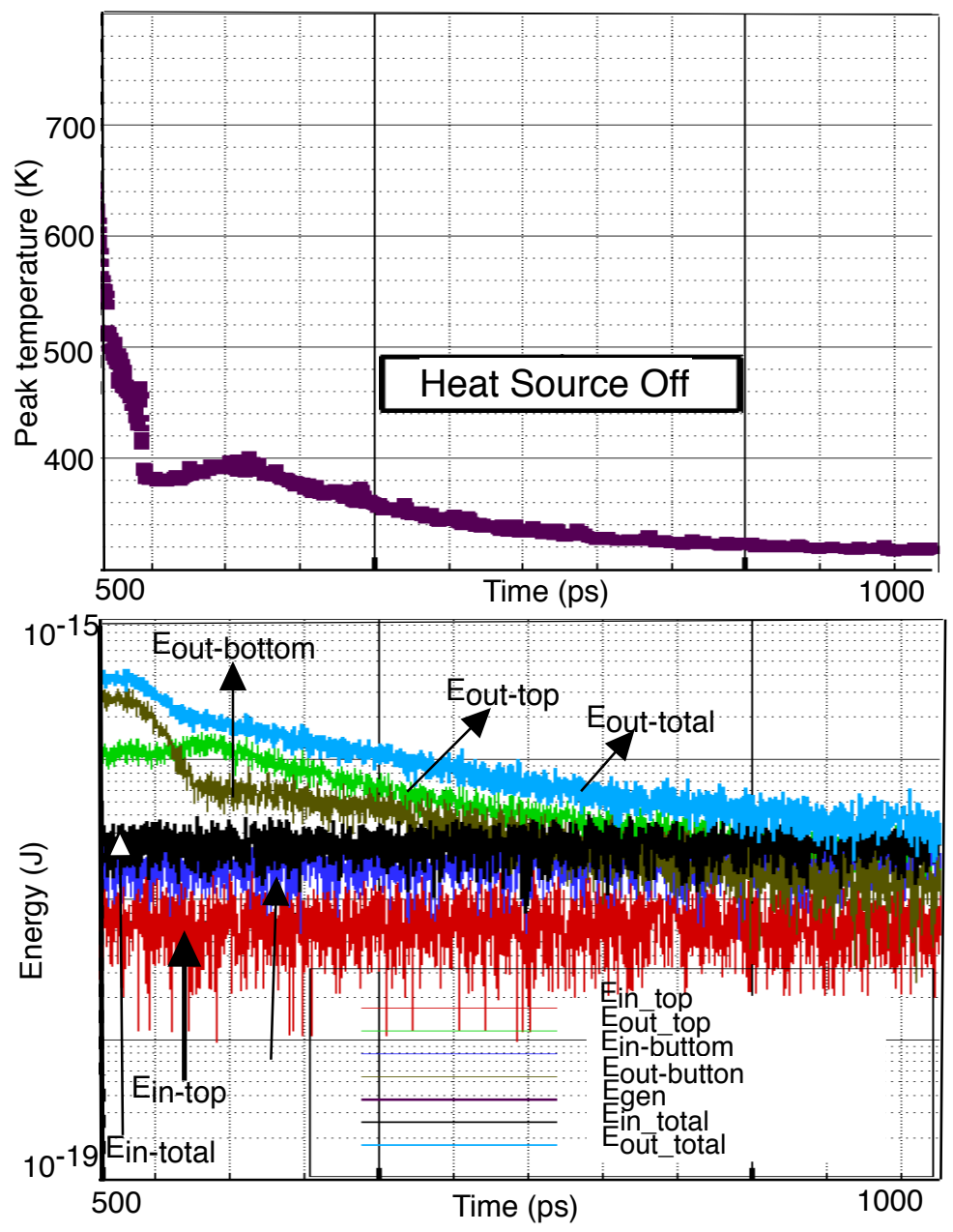

Figure 16: (a) The peak temperature rise versus time for the 3-D silicon transistor when the heating zone is switched off. (b) The energy trend in 3-D silicon transistor in bounding lines. 

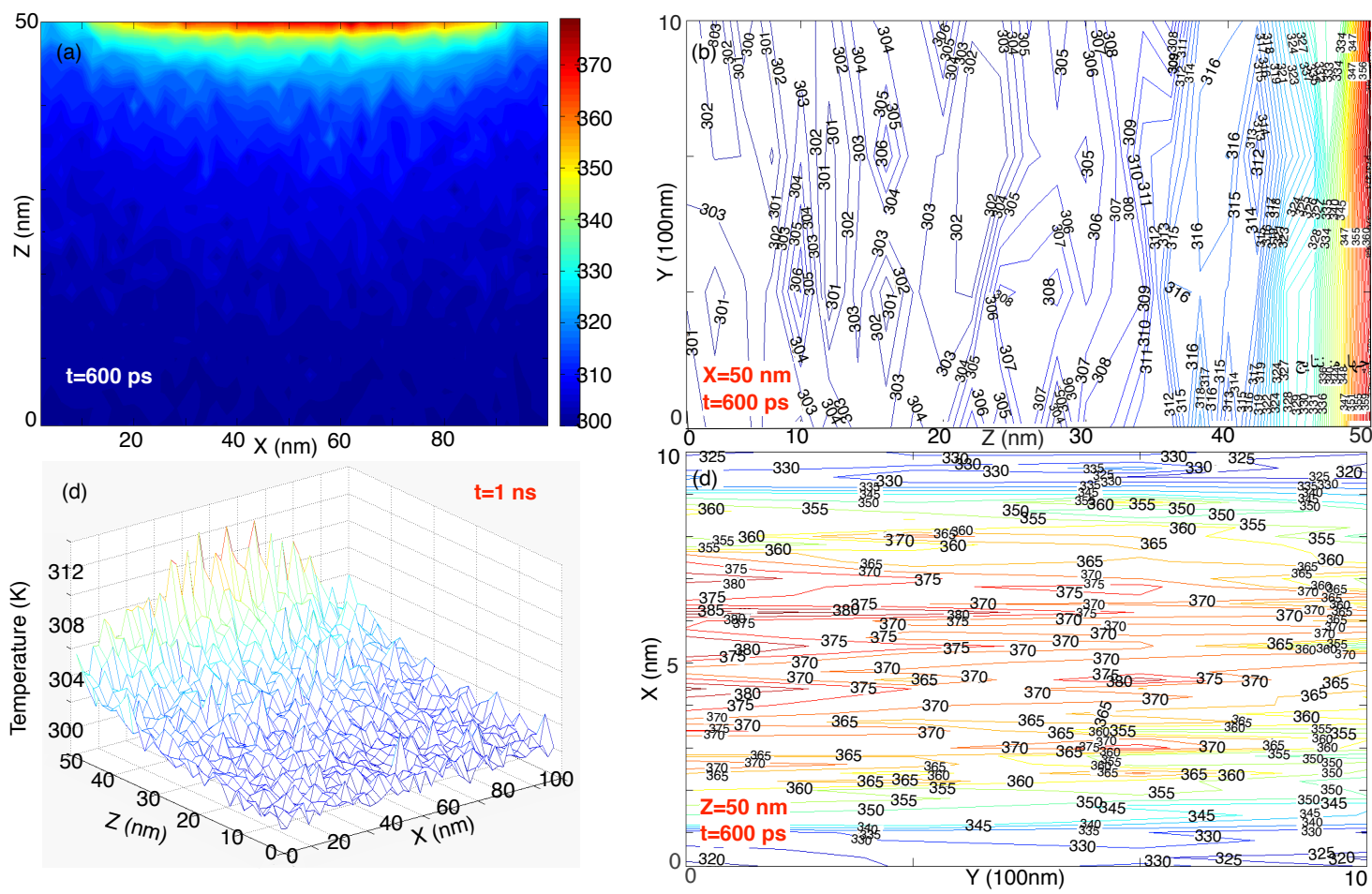

Figure 17: (a) The temperature profile averaged along the $Y$-direction in $X Z$ plane. (b) The temperature contour at $X=50 \mathrm{~nm}$ when $t=600 \mathrm{ps}$ in $Y Z$ plane. (c) The temperature distribution in $X Y$ plane at $Z=50 \mathrm{~nm}$ and $t=600 \mathrm{ps}$. (d) The 3-D plot of $Y$-averaged temperature versus $X$ and $Z$ at $t=1 \mathrm{~ns}$. 
757

temperature in the $X Y$ plane is demonstrated in Fig. 17(c) at $Z=50 \mathrm{~nm}$.

The figure exhibits that the minimum temperature is $320 \mathrm{~K}$, which is $20 \mathrm{~K}$ larger than the environment temperature. Further, it is shown that the temperature gradient along the $X$-direction on top of the MOSFET is very intense such that the heat transmission through this region is prominent. At last, the temperature distribution in $t=1 \mathrm{~ns}$ is plotted in $X Z$ plane. Fig. 17(d) displays this profile. The low temperature difference between the temperatures of the various places reveals the heat distribution in the MOSFET.

\section{Conclusions}

In this paper, the Monte-Carlo simulation of the three dimensional siliconbased MOSFET is performed. The accuracy of the numerical method is checked by verifying the obtained results with the existent data. Then, the 3-D MOSFET is studied under two different boundary conditions: (i) only the bottom boundary exchanges energy with the environment, and (ii) in addition to the bottom boundary, two top boundaries are also open to the surrounding. In both cases, the hot zone is formed. For the case one, the jump in the maximum temperature takes places due to the change in the type 
775

of the predominant heat carriers to the optical phonons. Moreover, many odd behaviours in the temperature profiles are found during the heating and cooling of the 3-D transistor. Also, it is obtained that the efficiency of the 3-D transistors by adding two top open boundaries, is improved. Although the new condition intensify the heat gathered in the hot-spot, it makes the heat spreading to the environment easier. In conclusion, the present work provides the exact data needed for the non-Fourier transient heat transfer analysis of multidimensional MOSFETs.

\section{Acknowledgement}

Zahra Shomali and Abbas Abbassi acknowledge support by the Iran National Science Foundation (INSF).

\section{Appendix}

At the first step of solving the Boltzmann equation, the actual number of phonons is obtained. Accordingly, the important parameter $W$ is calculated. This factor should be kept constant during the simulation. The actual number of phonons per volume for different branches in various temperatures are displayed in Fig. 18(a). Moreover, the phonon distribution in 
miscellaneous frequency intervals is highly non-linear. Hence, a cumulative density function is needed for ascribing the frequency to the phonon. This function is introduced in Eq. 7. Fig. 18(b) illustrates the behaviour of the number of phonons versus frequency. This figure illustrates that the most of the phonons belong to two main frequencies. The first one which has more phonons is assigned to the transitional phonons, and the second one is attributed to the optical phonons. Also, as it is seen in Fig. 18(c), using Eq. 7, and Fig. 18(b) the cumulative density function at different temperatures is obtained. It is obvious that when the temperature increases, the number of the optical phonons becomes larger. Besides, different branches are present in a specific frequency. This affects the type of the predominant phonons in heat transfer. To be more precise, at low temperatures the TA phonons are the main heat carriers in the MOSFET, but as the temperature enhances the presence of this branch fades out while the other kind of phonons appear. Fig. 18(d) shows the probability of the presence of each type of phonons in a peculiar frequency.

After that, the group velocity of different types of phonons is obtained by differentiation of the dispersion relations. Fig. 19, represents the comportment of the group velocity as a function of frequency. 

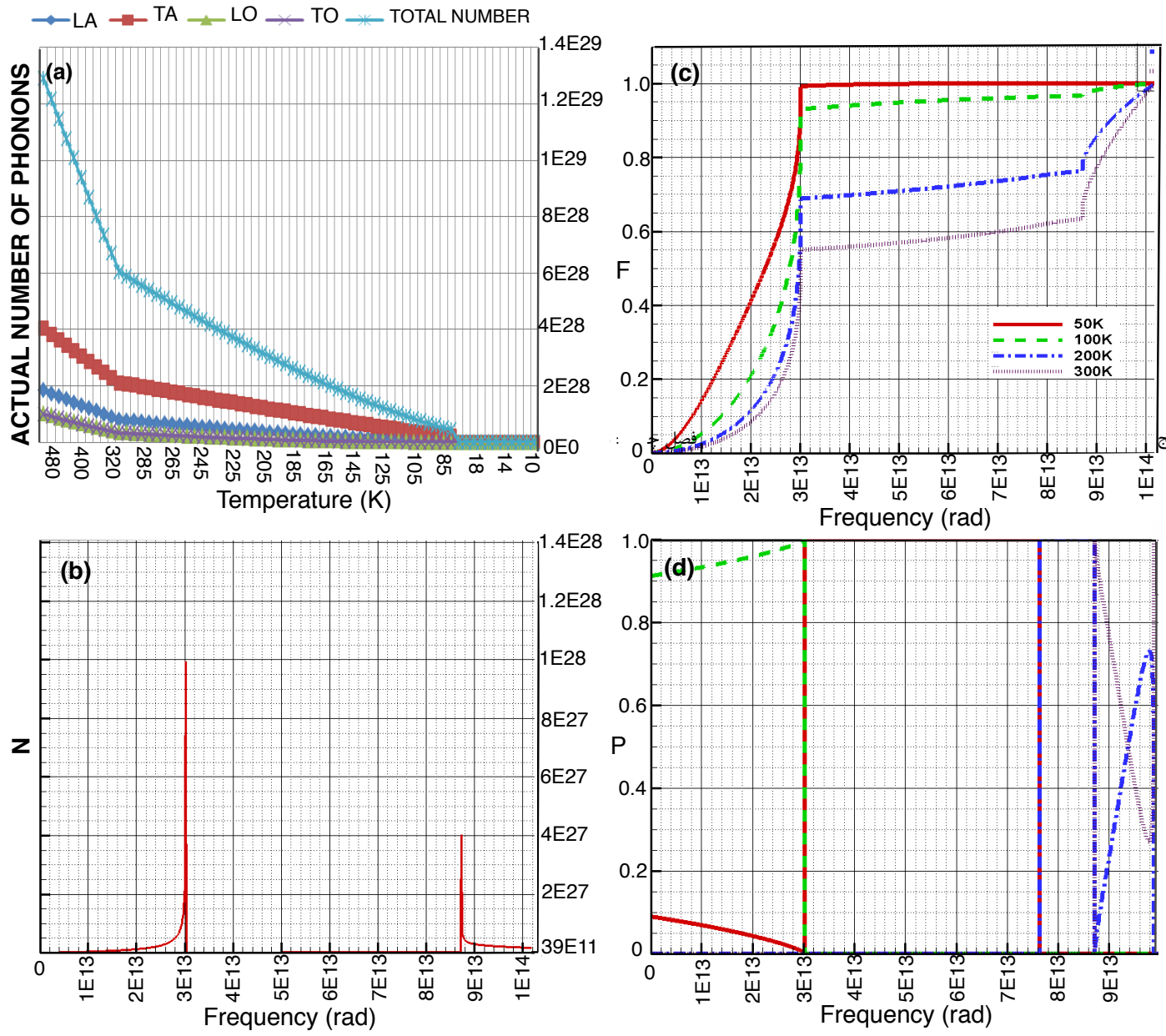

Figure 18: (a) The actual number of phonons per volume versus temperature for different branches. (b) The number of phonons as a function of frequency when $T=300 \mathrm{~K}$. (c) The cumulative phonon density of function. (d) The probability of the presence of the phonons in each branch. 


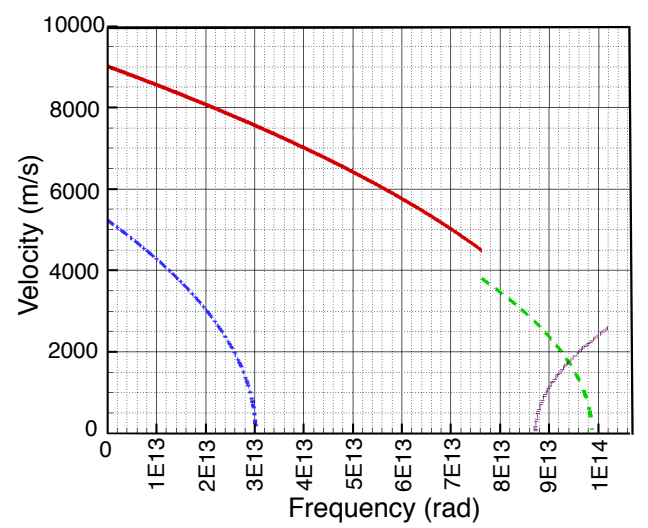

Figure 19: The group velocity of the various branches of $T A, L A, T O$, and $L O$ at $T=$ $300 \mathrm{~K}$.

At last, using the equations introduced in Sec. 3.6, the life-time of the phonons are calculated for different temperatures. As expected, the trend in Figs. 20(a-c) shows that the mean time between the scatterings decreases just when the temperature increases. This reduction is ascribed to the increment of the number of scatterings. Also, the obtained mean free path as an important parameter in the determination of heat transfer regime is shown in Fig. 20(d) when $T=300 \mathrm{~K}$.

\section{References}

[1] R.S. Samian, A. Abbassi, J. Ghazanfarian, Thermal Investigation of Common 2D FETs and New Generation of 3D FETs using Boltzmann Transport Equation in Nanoscale, Int. J. Mod Phys C, 2011, 24, 1350064. 

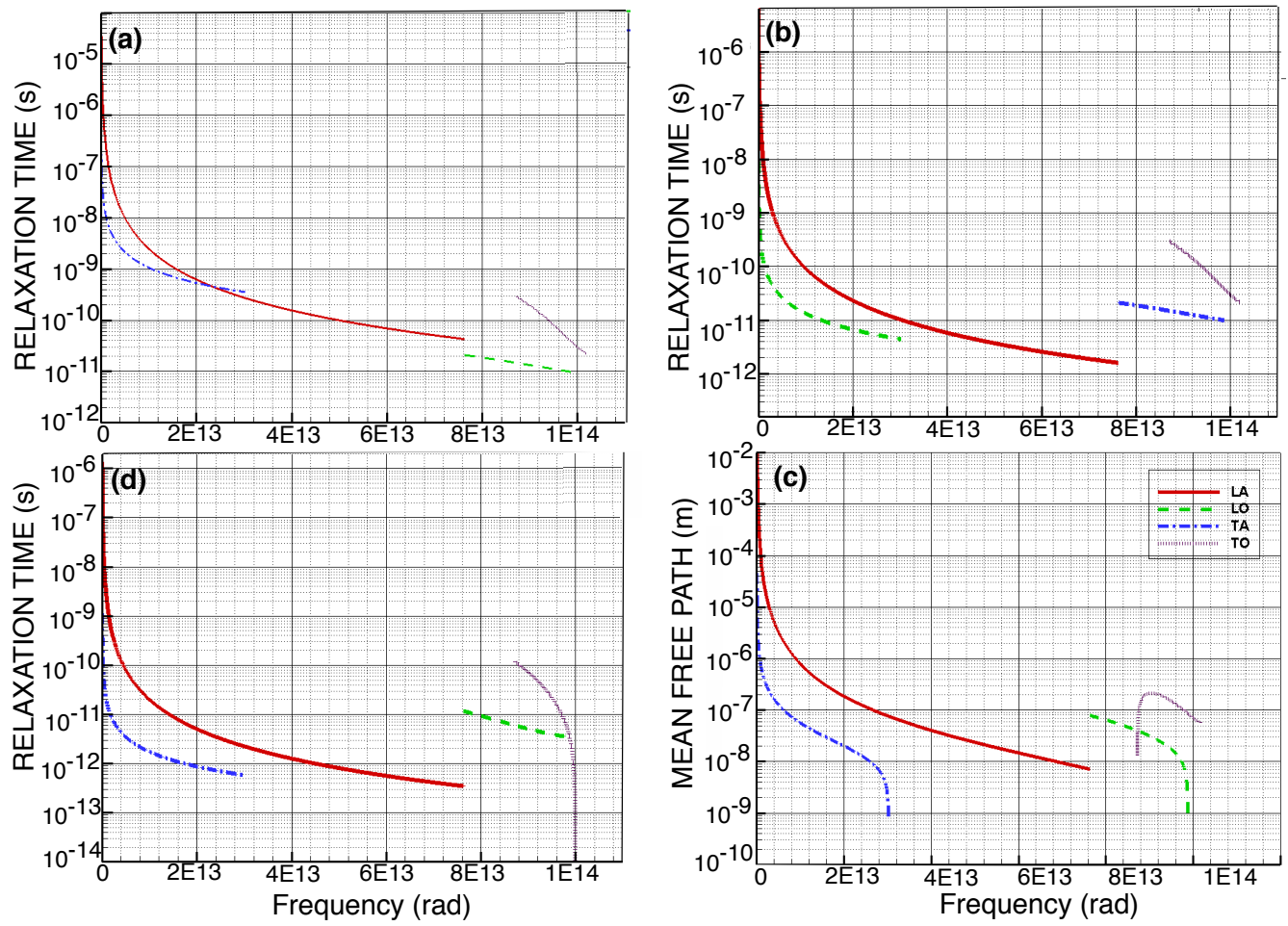

Figure 20: (a) The phonon life-time in different branches at $T=100 K$. (b) The same as (a) but when $T=300 \mathrm{~K}$. (c) The phonon relaxation time in $T=500 \mathrm{~K}$. (d) The phonon mean free path for $T A, L A, T O$ and $L O$ branches when $T=300 \mathrm{~K}$. 
[2] L..C. Liu, M.J. Huang, Thermal conductivity modeling of micro- and nanoporous silicon, Int. J. Thermal Sci., 2010, 49, 1547-1554.

[3] H.R. Ghazizadeh, A. Azimi, M. Maerefat, An inverse problem to estimate relaxation parameter and order of fractionality in fractional singlephase-lag heat equation, Int. J. Heat Mass Transfer, 2012, 55(7), 20952101.

[4] J. Ghazanfarian, A. Abbassi, Effect of boundary phonon scattering on Dual-Phase-Lag model to simulate micro- and nano-scale heat conduction, Int. J. Heat Mass Transfer, 2009, 52, 3706-3711.

[5] Z. Shomali, A. Abbassi, Investigation of highly non-linear dual-phase-lag model in nanoscale solid argon with temperature-dependent properties, Int. J. Therm. Sci. 2014, 87, 56.

[6] Z. Shomali, J. Ghazanfarian, A. Abbassi, Effect of film properties for non-linear DPL model in a nanoscale MOSFET with high-k material: $\mathrm{ZrO}_{2} / \mathrm{HfO}_{2} / \mathrm{La}_{2} \mathrm{O}_{3}$, Superlattices Microst., 2015, 83, 699.

[7] Y. Dong, B-Y. Cao, and Z-Y. Guo, Generalized heat conduction laws based on thermomass theory and phonon hydrodynamics, J. Appl Phys, 2011, 110(6), 063504. 
840

841

842

843

844

[8] M-J.. Huang, C-C. Weng, T-M. Chang, An investigation of the phonon properties of silicon nanowires, Int. J. Thermal Sci., 2010, 49, 1095-1102.

[9] J.P. Praud, N.G. Hadjiconstantinou, An alternative approach to efficient simulation of micro/nanoscale phonon transport Appl. Phys. Lett. 2012, 101(15), 153114.

[10] Y.C. Hua, B.Y. Cao, Phonon ballistic-diffusive heat conduction in silicon nanofilms by Monte Carlo simulations. Int. J. Heat Mass Transfer, 78, 755-759.

[11] S.A. Ali, G. Kollu, S. Mazumder, P. Sadayappan, A. Mittal, Large-scale parallel computation of the phonon Boltzmann Transport Equation, Int. J. Thermal Sci., 2010, 86, 341-351.

[12] Y.C. Hua, B.Y. Cao, Ballistic-diffusive heat conduction in multiplyconstrained nanostructures, Int. J. Thermal Sci., 2016, 101126-132.

[13] Y.C. Hua, B.Y. Cao, Transient in-plane thermal transport in nanofilms with internal heating, Proc. R. Soc. A, 2016, 472, 20150811; DOI: 10.1098/rspa.2015.0811. 
[14] D.S. Tang, Y.C. Hua, B.D. Nie, B.Y. Cao, Phonon wave propagation in ballistic-diffusive regime, J. App Phys, 2016, 119(12), 124301.

[15] M-J. Huang, T.Y. Kang, A Monte-Carlo study of the phonon transport in nanowire-embedded composites, Int. J. Thermal Sci., 2011, 50, 11561163.

[16] R.B. Peterson, Direct simulation of phonon-mediated heat transfer in a Debye crystal, J. Heat Transfer, 1994, 116, 815.

[17] S. Mazumder, A. Majumdar, Monte Carlo study of phonon transport in solid thin films including dispersion and polarization, J. Heat Transfer, 2001, 123, 749 .

[18] A.S. Majumdar, Microscale heat conduction in dielectric thin films, J. Heat Transfer, 1993, 115, 7.

[19] S.V.J. Narumanchi, J.Y. Murthy, and C.H. Amon, Submicron heat transport model in silicon accounting for phonon dispersion and polarization, J. Heat Transfer, 2004, 126, 946.

[20] T. Wang, Sub-Micron Thermal Transport in Ultra-scaled Metal-Oxide 
semiconductor devices, PhD thesis, School of Mechanical Engineering, Purdue University, 2007.

[21] A. Mittal, and S. Mazumder, Monte Carlo study of phonon heat conduction in silicon thin films including contributions of optical phonons, J. Heat Transfer, 2010, 132, 052402.

[22] B.T. Wong, M. Francoeur, M.P. Menguc, A Monte Carlo simulation for phonon transport within silicon structures at nanoscales with heat generation, Int. J. Heat Mass Transfer, 2011, 54.9, 1825.

[23] J. Randrianalisoa, D. Baillis, Monte Carlo simulation of steady-state microscale phonon heat transport, J. Heat Transfer, 2008, 130, 072404.

[24] N.G. Hadjiconstantinou, G.A. Radtke, L.L. Baker, On variance-reduced simulations of the Boltzmann transport equation for small-scale heat transfer applications, J. Heat Transfer, 2010, 132, 112401.

[25] Y. Chen, D. Li, J.R. Lukes, A. Majumdar, Monte Carlo simulation of silicon nanowire thermal conductivity, J. Heat Transfer, 2005, 127, 1129.

[26] D. Lacroix, K. Joulain, D. Terris, D. Lemonnier, Monte Carlo simulation of phonon confinement in silicon nanostructures: Application to the 
determination of the thermal conductivity of silicon nanowires, App. Phys. Lett., 2006, 89, 103104.

[27] Q. Hao, G. Chen, M-S. Jeng, Frequency-dependent Monte Carlo simulations of phonon transport in two-dimensional porous silicon with aligned pores, J. Appl. Phys., 2009,106, 114321.

[28] V. Jean, S. Fumeron, K. Termentzidis, S. Tutashkonko, D. Lacroix, Monte Carlo simulations of phonon transport in nanoporous silicon and germanium, J. Appl. Phys., 2014, 115, 024304.

[29] H. Hamzeh, F. Aniel, Monte Carlo study of phonon dynamics in III-V compounds, J. Appl. Phys., 2011, 109, 063511.

[30] A. Akturk, N. Goldsman, G. Metze, Self-consistent modeling of heating and MOSFET performance in 3-d integrated circuits, IEEE Trans. Electron Devices, 2005, 52, 2395.

[31] Z. Aksamija, U. Ravaioli, Joule heating and phonon transport in silicon MOSFETs, J. Comput. Electron, 2006, 5, 431.

[32] T. Sadi, R.W. Kelsall, N.J. Pilgrim, J-L. Thobel, F. Dessenne, Monte 
Carlo study of self-heating in nanoscale devices, J Comput Electron, $2012,11,118$.

[33] O. Muscato, W. Wagner, V. Di Stefano, Heat generation in silicon nanometric semiconductor devices, Int. J. Comput. Math. Electrical Electronic Engineering, 2014, 33, 1198.

[34] S. Mei, L.N. Maurer, Z. Aksamija, I. Knezevic, Full-dispersion Monte Carlo simulation of phonon transport in micron-sized graphene nanoribbons, J. Appl. Phys., 2014, 116, 164307.

[35] Q. Li, X. Zhu, Y. Xuan, Modeling heat generation in high power density nanometer scale GaAs/InGaAs/AlGaAs PHEMT, Int. J. Heat Mass Transfer, 2015, 81, 130.

[36] R. Yang, G. Chen, M. Laroche, Y. Taur, Simulation of nanoscale multidimensional transient heat conduction problems using ballistic-diffusive equations and phonon Boltzmann equation, ASME J. Heat Transfer 2005, 127, 298306.

[37] P.G. Sverdrup, Y.S. Ju, K.E. Goodson, Sub-continuum simulations of heat conduction in silicon-on-insulator transistors, J. Heat Transfer, 2001, 123, 130. 
[38] J. Ghazanfarian, Z. Shomali, Investigation of dual-phase-lag heat conduction model in a nanoscale metal-oxide-semiconductor field-effect transistor, Int. J. Heat Mass Transfer, 2012, 55, 6231.

[39] C. Kittel, Introduce to Solid State Physics, Wiley, New York (1996).

[40] A. Mittal, Prediction of Non-Equilibrium Heat Conduction in Crystalline Materials Using the Boltzmann Transport Equation for Phonons. PhD thesis, The Ohio State University, 2011.

[41] E. Pop, R.W. Dutton, K.E. Goodson, Analytic band Monte Carlo model for electron transport in Si including acoustic and optical phonon dispersion, J. Appl. Phys., 2004, 96(9), 4998-5005.

[42] A. Mittal, Monte-Carlo Study of Phonon Heat Conduction in Silicon Thin Films. MSc thesis, The Ohio State University, 2009.

[43] A. Lagae, P. Dutre. An efficient ray-quadrilateral intersection test, JGT, $2005,10,2332$.

[44] A. S. Henry, G. Chen, Spectral phonon transport properties of silicon based on molecular dynamics simulations and lattice dynamics, J. Comput. Theor. Nanosci, 2008, 5, 1. 
${ }_{940}[45]$ E. Pop, S. Sinha, K.E. Goodson, Heat generation and transport in nanometer-scale transistors, Proceedings of the IEEE,2006, 94, 1587. 